\title{
Stability of the inverse source problem for the Helmholtz equation in R3
}

\author{
Kirkeby, Adrian ; Henriksen, Mads T. R. ; Karamehmedovi, Mirza
}

\section{Published in:}

Inverse Problems

Link to article, DOI:

$10.1088 / 1361-6420 / a b 762 d$

Publication date:

2020

Document Version

Peer reviewed version

Link back to DTU Orbit

Citation (APA):

Kirkeby, A., Henriksen, M. T. R., \& Karamehmedovi, M. (2020). Stability of the inverse source problem for the

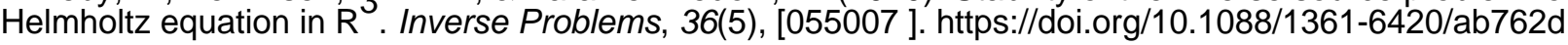

\section{General rights}

Copyright and moral rights for the publications made accessible in the public portal are retained by the authors and/or other copyright owners and it is a condition of accessing publications that users recognise and abide by the legal requirements associated with these rights.

- Users may download and print one copy of any publication from the public portal for the purpose of private study or research.

- You may not further distribute the material or use it for any profit-making activity or commercial gain

- You may freely distribute the URL identifying the publication in the public portal

If you believe that this document breaches copyright please contact us providing details, and we will remove access to the work immediately and investigate your claim. 
ACCEPTED MANUSCRIPT

\section{Stability of the inverse source problem for the Helmholtz equation in $<$ b $>$ R $</$ b $><$ sup $>3</$ sup $>$}

To cite this article before publication: Adrian Kirkeby et al 2020 Inverse Problems in press https://doi.org/10.1088/1361-6420/ab762d

\section{Manuscript version: Accepted Manuscript}

Accepted Manuscript is "the version of the article accepted for publication including all changes made as a result of the peer review process, and which may also include the addition to the article by IOP Publishing of a header, an article ID, a cover sheet and/or an 'Accepted Manuscript' watermark, but excluding any other editing, typesetting or other changes made by IOP Publishing and/or its licensors"

This Accepted Manuscript is @ 2020 IOP Publishing Ltd.

During the embargo period (the 12 month period from the publication of the Version of Record of this article), the Accepted Manuscript is fully protected by copyright and cannot be reused or reposted elsewhere.

As the Version of Record of this article is going to be / has been published on a subscription basis, this Accepted Manuscript is available for reuse under a CC BY-NC-ND 3.0 licence after the 12 month embargo period.

After the embargo period, everyone is permitted to use copy and redistribute this article for non-commercial purposes only, provided that they adhere to all the terms of the licence https://creativecommons.org/licences/by-nc-nd/3.0

Although reasonable endeavours have been taken to obtain all necessary permissions from third parties to include their copyrighted content within this article, their full citation and copyright line may not be present in this Accepted Manuscript version. Before using any content from this article, please refer to the Version of Record on IOPscience once published for full citation and copyright details, as permissions will likely be required. All third party content is fully copyright protected, unless specifically stated otherwise in the figure caption in the Version of Record.

View the article online for updates and enhancements. 


\title{
1 Introduction
}

Assume $R>R_{0}>0$ and let $B_{0}, B \subset \mathbf{R}^{3}$ be concentric open balls centered at the origin and of radius $R_{0}$ and $R$, respectively. Let $s \in L^{2}\left(B_{0}\right)$, and fix a positive frequency (wavenumber) $k$. Let $u_{k}$ be the unique outgoing solution of the Helmholtz equation in $\mathbf{R}^{3}$ with the inhomogeneous term $s$,

We have

\section{Abstract}

We consider the reconstruction of a compactly supported source term in the constantcoefficient Helmholtz equation in $\mathbf{R}^{3}$, from the measurement of the outgoing solution at a source-enclosing sphere. The measurement is taken at a finite number of frequencies. We explicitly characterize certain finite-dimensional spaces of sources that can be stably reconstructed from such measurements. The characterization involves only the measurement frequencies and the problem geometry parameters. We derive a singular value decomposition of the measurement operator, and prove a lower bound for the spectral bandwidth of this operator. By relating the singular value decomposition and the eigenvalue problem for the Dirichlet-Laplacian on the source support, we devise a fast and stable numerical method for the source reconstruction. We do numerical experiments to validate the stability and efficiency of the numerical method.

\section{Stability of the inverse source problem for the Helmholtz equation in $\mathbf{R}^{3}$}

\author{
Mads T. R. Henriksen* Mirza Karamehmedovićł and Adrian Kirkeby \\ Technical University of Denmark \\ Department of Mathematical Sciences $\ddagger$ \\ Trondheim, Norway
}

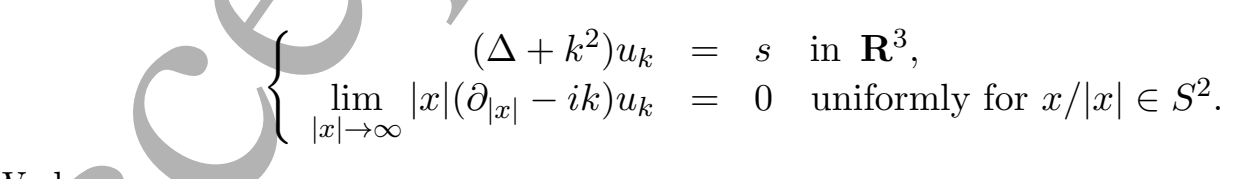

$$
u_{k}(x)=\int_{B_{0}} G_{k}(x-y) s(y) d y, \quad x \in \mathbf{R}^{3},
$$

${ }^{\dagger}$ mika@dtu.dk;note https://orcid.org/0000-0003-0038-9020

‡adrki@dtu.dk; https://orcid.org/0000-0003-2741-7423 
where $G_{k}$ is the outgoing fundamental solution of the Helmholtz equation in $\mathbf{R}^{3}$,

$$
G_{k}(x)=\frac{\exp (i k|x|)}{4 \pi|x|}, \quad x \in \mathbf{R}^{3} \backslash\{0\} .
$$

The forward operator, or measurement operator, $F_{k}$ associated with the frequency $k$ maps the source to the measurement, $F_{k} s=\left.u\right|_{\partial B}=U_{k}$. The mapping $F_{k}: L^{2}\left(B_{0}\right) \rightarrow L^{2}(\partial B)$ is linear and compact [5]. We call the trace $U_{k}$ the 'measurement at frequency $k$.' The inverse source problem (ISP) associated with (1) is:

Given the measurements $\left\{U_{k_{j}}=\left.u_{k_{j}}\right|_{\partial B}\right\}$ over a set of frequencies $Q=\left\{k_{j}\right\}$, estimate the source term $s$ in (1).

The multi-frequency ISP arises when the set $Q$ has more than one element.

The ISP for the Helmholtz equation in $\mathbf{R}^{3}$ appears, for example, in inverse acoustic scattering and linearized acoustic inverse obstacle and inverse medium problems. Physical applications of this include the design of sound sources with specified radiation patterns, submarine detection by sonar, reconstruction of seismic event history, nondestructive testing of materials, ocean bottom exploration for oil and gas, as well as medical imaging, for example in the form of photo- and thermoacoustic tomography. There is a vast mathematical, physical and engineering literature devoted to the study of the ISP and its applications; see, for instance, $[1,4,8,10,11,13,16$. Uniqueness, stability and inversion methods for the (multifrequency) ISP were treated, for example, in $[2,3,5,7,12,14,15,17,19,22,25$. The purpose of this work is to investigate the stability of the single-frequency and multi-frequency inverse source problem for the Helmholtz equation in $\mathbf{R}^{3}$, as well as to present a stable and effective reconstruction method for the multi-frequency problem. The central new technique in our reconstruction method is the explicit and mathematically justified procedure of choosing a sparse set of measurement frequencies that allows accurate solution, given some knowledge of the function space in which the source resides.

In section 2 we derive a singular value system of the forward operator $F_{k}$, and in section 3 we use this to give a non-asymptotic spectral characterization of $F_{k}$. The main result there is Theorem 2. The analysis in sections 2 and 3 follows our earlier work 14 in $\mathbf{R}^{2}$ rather closely. Section 4 concerns the multi-frequency ISP, and here we identify certain finite-dimensional spaces of sources that can be stably reconstructed from the boundary measurements. The main result there is Theorem 3, where we give a sufficient criterion for reconstruction of sources belonging to a class of finite-dimensional subspaces of $L^{2}\left(B_{0}\right)$. In section 5 we conduct numerical experiments to verify our analysis and the reconstruction method, and we finally offer our conclusions and suggestions for further work in section 6 .

\section{Remark}

Our analysis is applicable to more general measurement boundaries than the sphere, since in certain cases one may extend a measurement continuously to the sphere. More precisely, let $\Gamma$ be the boundary of some $C^{2}$ domain $\Omega$ that includes the source support $B_{0}$, and take $B$ to be the smallest ball that includes $\Omega$. Let $g=\left.u_{k}\right|_{\Gamma}$ be the measurement of the field $u_{k}$ on $\Gamma$. Then $g$ has a unique extension to a measurement $U_{k}$ on $\partial B$ that depends continuously on $g$ (cf. Theorem 3.11 in [8]). 


\section{$2 \quad$ A singular system of $F_{k}$}

Our results on the stability of solution of the multi-frequency inverse source problem rely on detailed spectral analysis of the measurement operator $F_{k}$. Thus, we first describe a singular value decomposition of $F_{k}$ in terms of spherical harmonics and spherical Bessel functions. Let $h_{m}^{(1)}$ be the spherical Hankel function of the first kind and order $m, j_{m}(z)$ the spherical Bessel function of order $m$, and $Y_{m}^{n}, m \in \mathbf{N}_{0}, n=-m, \ldots, m$, the spherical harmonics. Write $\kappa_{0}=k R_{0}$ and $\kappa=k R$, and let

$$
\begin{aligned}
a_{m}\left(\kappa_{0}\right) & =\frac{2 \sqrt{k / \pi}}{R_{0}}\left(\int_{0}^{R_{0}} j_{m}(k r)^{2} r^{2} d r\right)^{1 / 2}=\frac{2 \sqrt{k / \pi}}{R_{0}}\left(\int_{0}^{R_{0}} r^{2}\left(\sqrt{\frac{\pi}{2 k r}} J_{m+1 / 2}(k r)\right)^{2} \mathrm{~d} r\right)^{1 / 2} \\
& =\frac{\sqrt{2}}{R_{0}}\left(\int_{0}^{R_{0}} r J_{m+1 / 2}(k r)^{2} \mathrm{~d} r\right)^{1 / 2} \\
& =\left(J_{m+1 / 2}\left(\kappa_{0}\right)^{2}+J_{m-1 / 2}\left(\kappa_{0}\right)^{2}-\frac{2 m+1}{\kappa_{0}} J_{m+1 / 2}\left(\kappa_{0}\right) J_{m-1 / 2}\left(\kappa_{0}\right)\right)^{1 / 2} \\
& =\left(J_{m+1 / 2}\left(\kappa_{0}\right)^{2}+J_{m+3 / 2}\left(\kappa_{0}\right)^{2}-\frac{2 m+1}{\kappa_{0}} J_{m+1 / 2}\left(\kappa_{0}\right) J_{m+3 / 2}\left(\kappa_{0}\right)\right)^{1 / 2}
\end{aligned}
$$

where we use the well-known relation $j_{m}(z)=\sqrt{\pi / 2 z} J_{m+1 / 2}(z)$. The last equality in (4) follows after using the recurrence relation

$$
J_{m-1 / 2}\left(\kappa_{0}\right)=\frac{2(m+1 / 2)}{\kappa_{0}} J_{m+1 / 2}\left(\kappa_{0}\right)-J_{m+3 / 2}\left(\kappa_{0}\right) .
$$

For positive arguments, the function $j_{m}$ is real-valued and has zeros over a subset of the real axis with measure zero. Therefore, we have for any $m \in \mathbf{N}_{0}$ and any positive $\kappa_{0}$ that

$$
a_{m}\left(\kappa_{0}\right)=2 \sqrt{k / \pi} R_{0}^{-1}\left(\int_{0}^{R_{0}} j_{m}(k r)^{2} r^{2} d r\right)^{1 / 2}>0 .
$$

Theorem 1. The measurement operator $F_{k}$ admits the singular value decomposition

with

$$
F_{k}=\sum_{m \in \mathbf{N}_{0}} \sum_{n=-m}^{m} \sigma_{m}^{k}\left(\cdot, u_{m, n}^{k}\right)_{L^{2}\left(B_{0}\right)} v_{m, n}^{k}
$$

The $m$ 'th singular value $\sigma_{m}^{k}$ has the multiplicity $2 m+1$. 
Proof. The fundamental solution (3) of the Helmholtz equation in $\mathbf{R}^{3}$ has an expansion in terms of the spherical harmonics [8, Theorem 2.11, page 31],

$$
G_{k}(x-y)=i k \sum_{m=0}^{\infty} \sum_{n=-m}^{m} h_{m}^{(1)}(k|x|) Y_{m}^{n}(x /|x|) j_{m}(k|y|) \overline{Y_{m}^{n}(y /|y|)}, \quad|x|>|y| .
$$

The $L^{2}$-adjoint $F_{k}^{*}: L^{2}(\partial B) \rightarrow L^{2}\left(B_{0}\right)$ of $F_{k}$ is given by

$$
F_{k}^{*} U(x)=\int_{\partial B} \overline{G_{k}(x-y)} U(y) \mathrm{d} S(y), \quad U \in L^{2}(\partial B), \quad x \in B_{0} .
$$

Since $\overline{B_{0}} \subset B$, we have $|z|>|x|$ whenever $z \in \partial B$ and $x \in B_{0}$. Also, $\left\{Y_{m}^{n}\right\}$ is an orthonormal basis of $L^{2}\left(S^{2}\right)$, and $G_{k}(x-y)=G_{k}(y-x)$, so for every $s \in L^{2}\left(B_{0}\right)$ and $x \in B_{0}$ it holds that

$$
\begin{aligned}
F_{k}^{*} F_{k} s(x) & =\int_{\partial B} \overline{G_{k}(z-x)} \int_{B_{0}} G_{k}(z-y) s(y) \mathrm{d} y \mathrm{~d} S(z) \\
& =\int_{B_{0}} s(y) \int_{\partial B} \overline{G_{k}(z-x)} G_{k}(z-y) \mathrm{d} S(z) \mathrm{d} y \\
& =-\int_{B_{0}} s(y) \int_{\partial B} i k \sum_{m=0}^{\infty} \sum_{n=-m}^{m} \overline{h_{m}^{(1)}(k|z|) Y_{m}^{n}(z /|z|) j_{m}(k|x|)} Y_{m}^{n}(x /|x|) \\
& \times i k \sum_{\mu=0}^{\infty} \sum_{\nu=-\mu}^{\mu} h_{\mu}^{(1)}(k|z|) Y_{\mu}^{\nu}(z /|z|) j_{\mu}(k|y|) \overline{Y_{\mu}^{\nu}(y /|y|)} \mathrm{d} S(z) \mathrm{d} y \\
& =\kappa^{2} \sum_{m=0}^{\infty} \sum_{n=-m}^{m}\left|h_{m}^{(1)}(\kappa)\right|^{2} \bar{j}_{m}(k|x|) Y_{m}^{n}(x /|x|) \int_{B_{0}} s(y) j_{m}(k|y|) \overline{Y_{m}^{n}(y /|y|)} \mathrm{d} y .
\end{aligned}
$$

This is, in fact, an eigendecomposition of the operator $F_{k}^{*} F_{k}$,

$$
F_{k}^{*} F_{k}=\sum_{m \in \mathbf{N}_{0}} \sum_{n=-m}^{n}\left(\sigma_{m, n}^{k}\right)^{2}\left(\cdot, u_{m, n}^{k}\right)_{L^{2}\left(B_{0}\right)} u_{m, n}^{k}
$$

with (normalized) eigenvectors

$$
u_{m, n}^{k}(x)=\frac{j_{m}(k|x|) Y_{m}^{n}(x /|x|)}{\left\|j_{m}(k|x|) Y_{m}^{n}(x /|x|)\right\|_{L^{2}\left(B_{0}\right)}}, \quad x \in B_{0}, m \in \mathbf{N}_{0}, n=-m, \ldots, m,
$$

and eigenvalues

$$
\left(\sigma_{m, n}^{k}\right)^{2}=\kappa^{2}\left|h_{m}^{(1)}(\kappa)\right|^{2}\left\|j_{m}(k|x|) Y_{m}^{n}(x /|x|) \mid\right\|_{L^{2}\left(B_{0}\right)}^{2}, \quad m \in \mathbf{N}_{0}, n=-m, \ldots, m .
$$

Now each spherical harmonic $Y_{m}^{n}$ has unit norm in $L^{2}\left(S^{2}\right)$, so

$$
\begin{aligned}
\left\|j_{m}(k|x|) Y_{m}^{n}(x /|x|) \mid\right\|_{L^{2}\left(B_{0}\right)}^{2} & =\int_{B_{0}}\left|j_{m}(k|x|) Y_{m}^{n}(x /|x|)\right|^{2} \mathrm{~d} x \\
& =\int_{0}^{R_{0}} \int_{0}^{2 \pi} \int_{0}^{\pi} j_{m}(k r)^{2}\left|Y_{m}^{n}(\theta, \varphi)\right|^{2} r^{2} \sin \theta \mathrm{d} \theta \mathrm{d} \varphi \mathrm{d} r \\
& =\int_{0}^{R_{0}} r^{2} j_{m}(k r)^{2} \mathrm{~d} r=\frac{R_{0}^{2} \pi}{4 k} a_{m}\left(\kappa_{0}\right)^{2}
\end{aligned}
$$


Hence

$$
u_{m, n}^{k}(x)=\frac{2 \sqrt{k / \pi}}{R_{0} a_{m}\left(\kappa_{0}\right)} j_{m}(k|x|) Y_{m}^{n}(x /|x|), \quad x \in B_{0}, m \in \mathbf{N}_{0}, n=-m, \ldots, m
$$

and

$$
\left(\sigma_{m, n}^{k}\right)^{2}=\frac{k R^{2} R_{0}^{2} \pi}{4}\left|h_{m}^{(1)}(\kappa)\right|^{2} a_{m}\left(\kappa_{0}\right)^{2}, \quad m \in \mathbf{N}_{0}, n=-m, \ldots, m
$$

In particular, the eigenvalues $\left(\sigma_{m, n}^{k}\right)^{2}$ of $F_{k}^{*} F_{k}$ are degenerate with the degree of degeneracy $2 m+1$. In the following, we write $\left(\sigma_{m}^{k}\right)^{2}$ for these eigenvalues. By definition, the operator $F_{k}$ admits the singular values $\sigma_{m}^{k}$, as in (6). By Theorem 4.7 and its proof in [8, pp. 100-101] we have the associated right-singular vectors and left-singular vectors of $F_{k}$ given respectively by $u_{m, n}^{k}$, as in (7), and by

$$
\begin{aligned}
v_{m, n}^{k}(x) & =\frac{1}{\sigma_{m, n}^{k}} F_{k} u_{m, n}^{k}(x)=\frac{4}{\pi R_{0}^{2} R\left|h_{m}^{(1)}(\kappa)\right| a_{m}\left(\kappa_{0}\right)^{2}} \int_{B_{0}} G_{k}(x-y) j_{m}(k|y|) Y_{m}^{n}(y /|y|) \mathrm{d} y \\
& =\frac{4 \mathrm{i} k}{\pi R_{0}^{2} R\left|h_{m}^{(1)}(\kappa)\right| a_{m}\left(\kappa_{0}\right)^{2}} \sum_{\mu=0}^{\infty} \sum_{\nu=-\mu}^{\mu} h_{\mu}^{(1)}(\kappa) Y_{\mu}^{\nu}(x /|x|) \int_{B_{0}} j_{m}(k|y|) j_{\mu}(k|y|) Y_{m}^{n}(y /|y|) \overline{Y_{\mu}^{\nu}(y /|y|)} \mathrm{d} y \\
& =\frac{4 \mathrm{i} k \exp \left(\mathrm{i} \arg h_{m}^{(1)}(\kappa)\right)}{\pi R_{0}^{2} R a_{m}\left(\kappa_{0}\right)^{2}} Y_{m}^{n}(x /|x|) \int_{r=0}^{R_{0}} r^{2} j_{m}(k r)^{2} \mathrm{~d} r \\
& =\frac{\mathrm{i} \exp \left(\mathrm{i} \arg h_{m}^{(1)}(\kappa)\right)}{R} Y_{m}^{n}(x /|x|), \quad x \in \partial B,
\end{aligned}
$$

as in (8).

Remark 1. In [14, Lemma 1, p. 4] we followed Bao, Lin and Triki [5] and found the following expression for singular values of the measurement operator for the Helmholtz equation in the plane, at frequency $k$ :

$$
\sigma_{2 \mathrm{D}, m}^{k}=\sqrt{2 R} \pi R_{0}\left|H_{m}^{(1)}(\kappa)\right| A_{m}\left(\kappa_{0}\right), \quad m \in \mathbf{N}_{0} .
$$

Here $H_{m}^{(1)}=J_{m}+\mathrm{i} Y_{m}$ is the Hankel function of the first kind and order $m, Y_{m}$ is the Bessel function of the second kind (also called the Neumann function) of order $m$, and

$$
A_{m}\left(\kappa_{0}\right)=\left(J_{m}\left(\kappa_{0}\right)^{2}+J_{m+1}\left(\kappa_{0}\right)^{2}-\frac{2 m}{\kappa_{0}} J_{m}\left(\kappa_{0}\right) J_{m+1}\left(\kappa_{0}\right)\right)^{1 / 2}, \quad m \in \mathbf{N}_{0} .
$$

Since the Bessel functions are defined for all real orders, we may extend the expression for $\sigma_{2 \mathrm{D}, m}^{k}$ to $m>0$ and use the relation $h_{m}^{(1)}(z)=\sqrt{\pi / 2 z} H_{m+1 / 2}^{(1)}(z)$ to express the singular values (6) by

$$
\sigma_{m}^{k}=\frac{1}{4} \sigma_{2 \mathrm{D}, m+1 / 2}^{k}, \quad m \in \mathbf{N}_{0} .
$$

\section{Spectral bandwidth of $F_{k}$}

As we did in [14, we here define the bandwidth $\mathscr{B}$ of the operator $F_{k}: L^{2}\left(B_{0}\right) \rightarrow L^{2}(\partial B)$ to be

$$
\mathscr{B}=\operatorname{argmin}_{m \in \mathbf{N}_{0}}\left\{\sigma_{m+n}^{k}>\sigma_{m+n+1}^{k} \text { for all } n \in \mathbf{N}_{0}\right\},
$$


that is, the smallest singular value index after which the singular values, ordered as in Theorem 1, are strictly decaying. In [14] we proved and validated numerically an explicit tight lower bound for the bandwidth of $F_{k}$ in $\mathbf{R}^{2}$, as well as argued analytically and numerically for a conjectured tight upper bound. In [15] we used the lower bound from 14 to ensure stability of solution of the multi-frequency problem in $\mathbf{R}^{2}$. We now obtain a lower bound for the bandwidth $\mathscr{B}$ of $F_{k}$ in $\mathbf{R}^{3}$ analogous to that in the two-dimensional case. First we need a technical result; let $m \in \mathbf{N}_{0}$.

Lemma 1. If $j_{\xi}\left(\kappa_{0}\right)=0$ for some $\xi \in[m, m+1]$ then $a_{m}\left(\kappa_{0}\right) \leq a_{m+1}\left(\kappa_{0}\right)$.

Proof. Since $j_{m}(z)=\sqrt{\pi / 2 z} J_{m+1 / 2}(z)$, we have from (4) that

$$
\begin{aligned}
a_{m}\left(\kappa_{0}\right) & =\sqrt{J_{m+1 / 2}\left(\kappa_{0}\right)^{2}+J_{m-1 / 2}\left(\kappa_{0}\right)^{2}-\frac{2 m+1}{\kappa_{0}} J_{m+1 / 2}\left(\kappa_{0}\right) J_{m-1 / 2}\left(\kappa_{0}\right)} \\
& =\sqrt{\frac{2 \kappa_{0}}{\pi}} \sqrt{j_{m}\left(\kappa_{0}\right)^{2}+j_{m-1}\left(\kappa_{0}\right)^{2}-\frac{2 m+1}{\kappa_{0}} j_{m}\left(\kappa_{0}\right) j_{m-1}\left(\kappa_{0}\right) .}
\end{aligned}
$$

Omitting, for simplicity, the arguments $\left(\kappa_{0}\right)$, and using the well-known recurrence relation

$$
j_{m-1}+j_{m+1}=\sqrt{\frac{\pi}{2 \kappa_{0}}}\left(J_{m-1 / 2}+J_{m+3 / 2}\right)=\sqrt{\frac{\pi}{2 \kappa_{0}}} \frac{2(m+1 / 2)}{\kappa_{0}} J_{m+1 / 2}=\frac{2 m+1}{\kappa_{0}} j_{m},
$$

we find

$$
\begin{aligned}
\frac{\pi}{2 \kappa_{0}}\left(a_{m}^{2}-a_{m+1}^{2}\right) & =j_{m-1}^{2}-j_{m+1}^{2}-\frac{2 m+1}{\kappa_{0}} j_{m} j_{m-1}+\frac{2 m+3}{\kappa_{0}} j_{m} j_{m+1} \\
& =\left(j_{m-1}-j_{m+1}\right)\left(j_{m-1}+j_{m+1}\right)-\frac{2 m+1}{\kappa_{0}} j_{m}\left(j_{m-1}-j_{m+1}\right)+\frac{2}{\kappa_{0}} j_{m} j_{m+1} \\
& =\frac{2 m+1}{\kappa_{0}} j_{m}\left(j_{m-1}-j_{m+1}\right)-\frac{2 m+1}{\kappa_{0}} j_{m}\left(j_{m-1}-j_{m+1}\right)+\frac{2}{\kappa_{0}} j_{m} j_{m+1} \\
& =\frac{2}{\kappa_{0}} j_{m} j_{m+1} .
\end{aligned}
$$

It was shown in Lemma 2 in 14 that if $\mu_{2}>\mu_{1}>0$ and $J_{\mu_{1}}\left(\kappa_{0}\right)=J_{\mu_{2}}\left(\kappa_{0}\right)=0$ then $\mu_{2}-\mu_{1}>1$. This result implies that if $\mu_{2}>\mu_{1}>0$ and $j_{\mu_{1}}\left(\kappa_{0}\right)=j_{\mu_{2}}\left(\kappa_{0}\right)=0$ then $\mu_{2}-\mu_{1}>1$. Thus, if $j_{\xi}\left(\kappa_{0}\right)=0$ for some $\xi \in[m, m+1]$ then the function $\mathbf{R} \ni \mu \mapsto j_{\mu}\left(\kappa_{0}\right)$ equals zero at $m$, or it equals zero at $m+1$, or it changes sign exactly once in the interval $[m, m+1]$. In either case, we have

$$
a_{m}^{2}-a_{m+1}^{2}=\frac{4}{\pi} j_{m} j_{m+1} \leq 0
$$

and it remains to recall that $a_{m}\left(\kappa_{0}\right)>0$ for all $m \in \mathbf{N}_{0}$ and $\kappa_{0}>0$.

Lemma 2. The function $m \mapsto\left|h_{m}^{(1)}(\kappa)\right|$ is strictly increasing for $m>-1 / 2$ and $\kappa>0$.

Proof. The argument is completely analogous to that in Remark 1 in [14. By Nicholson's integral theorem [23, pp. 441-444], we have

$$
\begin{aligned}
\left.1(\kappa)\right|^{2} & =\left|j_{m}(\kappa)+i y_{m}(\kappa)\right|^{2}=j_{m}(\kappa)^{2}+y_{m}(\kappa)^{2} \\
& =\frac{\pi}{2 \kappa}\left(J_{m+1 / 2}(\kappa)^{2}+Y_{m+1 / 2}(\kappa)^{2}\right)=\frac{\pi}{2 \kappa}\left|H_{m+1 / 2}^{(1)}(\kappa)\right|^{2} \\
& =\frac{4}{\pi \kappa} \int_{0}^{\infty} \cosh \left(2\left(m+\frac{1}{2}\right) t\right) K_{0}(2 \kappa \sinh t) d t
\end{aligned}
$$


where $K_{0}$ is the modified Bessel function of the second kind. Differentiating w.r.t. order,we get

$$
\partial_{m}\left|h_{m}^{(1)}(\kappa)\right|^{2}=\frac{8}{\pi \kappa}\left(m+\frac{1}{2}\right) \int_{0}^{\infty} \sinh \left(2\left(m+\frac{1}{2}\right) t\right) K_{0}(2 \kappa \sinh t) d t
$$

and since $K_{0}$ and the hyperbolic sine are positive for positive arguments, it holds that

$$
\partial_{m}\left|h_{m}^{(1)}(\kappa)\right|^{2}>0 \quad \text { for all } m>-1 / 2
$$

This implies that $\left|h_{m}^{(1)}(\kappa)\right|$ is strictly increasing for $m>-1 / 2$ and $\kappa>0$.

For $m \geq 0$ and $n \in \mathbf{N}$, let $j_{m+1 / 2, n}$ be the $n$ 'th positive zero of the Bessel function $J_{m+1 / 2}$, that is, the $n$ 'th positive zero of the spherical Bessel function $j_{m}$.

Theorem 2. A lower bound $\mathscr{B}_{-}$for $\mathscr{B}$ is given by $\mathscr{B}_{-}=\operatorname{argmin}_{m \in \mathbf{N}_{0}}\left\{j_{m+1 / 2,1} \geq \kappa_{0}\right\}$.

Proof. Let $m \in \mathbf{N}_{0}$. If $j_{m+1 / 2,1}<\kappa_{0}$ then, due to the well-known continuity and strictly increasing behavior of the function $\mu \mapsto j_{\mu, 1}$, the value $\kappa_{0}$ is the first positive zero of some Bessel function $J_{\xi+1 / 2}$ with $\xi>m$, hence it is the first positive zero of some spherical Bessel function $j_{\xi}$ with $\xi>m$. There are thus $n \in \mathbf{N}_{0}$ and $\xi \in[m+n, m+n+1]$ satisfying $j_{\xi}\left(\kappa_{0}\right)=0$, and, by lemma 1, we have $a_{m+n}\left(\kappa_{0}\right) \leq a_{m+n+1}\left(\kappa_{0}\right)$. Also, by lemma 2 the function $\mathbf{N}_{0} \ni \mu \mapsto\left|h_{\mu}^{(1)}(\kappa)\right|$ is strictly increasing. Since the singular value $\sigma_{\mu}$ from (6) is proportional to $\left|h_{\mu}^{(1)}(\kappa)\right| a_{\mu}\left(\kappa_{0}\right)$ for $\mu \in \mathbf{N}_{0}$, we have $\sigma_{m+n} \leq \sigma_{m+n+1}$, hence $m<\mathscr{B}$. In conclusion,

In [14] we found a lower bound $\mathscr{B}_{2 D,-}$ for the two-dimensional case to be

$$
\mathscr{\mathscr { B }}_{2 D,-}=\operatorname{argmin}_{m \in \mathbf{N}_{0}}\left\{j_{m, 1} \geq \kappa_{0}\right\} .
$$

In light of the strictly increasing behavior of the function $\mu \mapsto j_{\mu, 1}$ for nonnegative orders $\mu$, we interpret the additional ' $+1 / 2$ ' in the order of the zero in the three-dimensional case as an indication of greater stability of the inverse source problem in $\mathbf{R}^{2}$ compared to the three-dimensional case. However, other aspects of the change of setting from $\mathbf{R}^{2}$ to $\mathbf{R}^{3}$ may offset this. We finally note that it might be possible to exploit the simple connection (11) between the singular values of the measurement operator in $\mathbf{R}^{2}$ and in $\mathbf{R}^{3}$ in an analysis along the lines of [14,pp. 9-10] and potentially obtain estimates of tight upper bounds on the bandwidths of both near-field and far-field measurement operators in $\mathbf{R}^{3}$. We defer this to future work, in particular since it suffices to use the lower bound $\mathscr{B}_{-}$in our stability analysis of the multi-frequency inverse source problem below.

With the singular value decomposition and bandwidth results for the measurement operator in place, we begin the analysis of the multi-frequency inverse source problem. 


\section{Stability of the multi-frequency inverse source problem in $\mathbf{R}^{3}$}

It is well-known [2] that the multi-frequency inverse source problem, as described in our introduction and in [2, Problem 3.1], is uniquely solvable only if measurements are available over a set of frequencies with an accumulation point, possibly at infinity. In practical settings, it is impossible to perform infinitely many measurements, so we turn to reconstructing sources in finite-dimensional subspaces of $L^{2}\left(B_{0}\right)$. The analysis in this section is a natural extension of the methods in 15 from $\mathbf{R}^{2}$ to $\mathbf{R}^{3}$, formulated in a simpler and more flexible way.

\subsection{Finite-dimensional source spaces}

Consider first the eigenvalue problem for the Dirichlet-Laplacian in $B_{0}$,

$$
\begin{aligned}
-\Delta \varphi & =\lambda \varphi \quad \text { in } B_{0}, \\
\varphi & =0 \quad \text { on } \partial B_{0} .
\end{aligned}
$$

One can check 20] that the eigenfunctions and eigenvalues are given uniquely up to a scaling by

$$
\begin{aligned}
& \varphi_{\alpha, \beta, \gamma}=\sqrt{\frac{2}{R_{0}^{3}}} \frac{j_{\alpha}\left(\mu_{\alpha, \beta}|x| / R_{0}\right)}{\left|j_{\alpha+1}\left(\mu_{\alpha, \beta}\right)\right|} Y_{\alpha}^{\gamma}(x /|x|), \quad \alpha \in \mathbf{N}_{0}, \beta \in \mathbf{N}, \gamma \in\{-\alpha, \ldots, \alpha\}, \\
& \lambda_{\alpha, \beta}=\left(\frac{\mu_{\alpha, \beta}}{R_{0}}\right)^{2}, \quad \alpha \in \mathbf{N}_{0}, \beta \in \mathbf{N} \text {, }
\end{aligned}
$$

where $\mu_{\alpha, \beta}$ is the $\beta$ 'th positive zero of the spherical Bessel function of order $\alpha$, i.e., $j_{\alpha}\left(\mu_{\alpha, \beta}\right)=$ 0 for $\beta \in \mathbf{N} 1$. Each eigenvalue $\lambda_{\alpha, \beta}$ has multiplicity $2 \alpha+1$.

It is well-known that the set $\left\{\varphi_{\alpha, \beta, \gamma}\right\}$ of normalized eigenfunctions is an orthonormal basis of $L^{2}\left(B_{0}\right)$. Any source $s \in L^{2}\left(B_{0}\right)$ thus has an eigenfunction expansion

$$
s(x)=\sum_{\alpha, \beta, \gamma} \hat{s}_{\alpha, \beta, \gamma} \varphi_{\alpha, \beta, \gamma}(x), \quad \hat{s}_{\alpha, \beta, \gamma}=\int_{B_{0}} s \bar{\varphi}_{\alpha, \beta, \gamma} \mathrm{d} x .
$$

For $M \in \mathbf{N}$, let $\Lambda_{M}=\left\{\lambda_{\alpha, \beta}\right\}$ be a set of any $M$ eigenvalues in (15). We associate with $\Lambda_{M}$ the finite-dimensional source space

$$
S_{\Lambda_{M}}\left(B_{0}\right)=\operatorname{span}\left\{\varphi_{\alpha, \beta, \gamma}: \lambda_{\alpha, \beta} \in \Lambda_{M},-\alpha \leq \gamma \leq \alpha\right\} \subset L^{2}\left(B_{0}\right) .
$$

Due to the multiplicity of the eigenvalues, it follows that $M \leq \operatorname{dim} S_{\Lambda_{M}}\left(B_{0}\right) \leq M(1+2 A)$, with $A=\max \left\{\alpha, \lambda_{\alpha, \beta} \in \Lambda_{M}\right\}$. A natural example of such finite-dimensional source space is given by the sources of the form

$$
s=\sum_{\alpha=0}^{N_{1}} \sum_{\beta=1}^{N_{2}} \sum_{\gamma=-\alpha}^{\alpha} \hat{s}_{\alpha, \beta, \gamma} \varphi_{\alpha, \beta, \gamma} .
$$

Then $\Lambda_{M}=\left\{\lambda_{\alpha, \beta}\right\}_{\substack{\alpha=0, \ldots, N_{1} \\ \beta=1, \ldots, N_{2}}}$, written $\left\{\lambda_{\alpha, \beta}\right\}_{\alpha=0, \beta=1}^{N_{1}, N_{2}}$ in the following, and hence $M=N_{2}\left(N_{1}+1\right)$ and $\operatorname{dim} S_{\Lambda_{M}}\left(B_{0}\right)=N_{2}\left(N_{1}+1\right)^{2}$.

\footnotetext{
${ }^{1}$ For clarity we here use the notation $\mu_{\alpha, \beta}=j_{\alpha+1 / 2, \beta}$.
} 
As we will see, the spaces $S_{\Lambda_{M}}\left(B_{0}\right)$ allow us to relate in a constructive manner the source information contained in the measurements at different frequencies to a common basis. We now address the following questions.

1. Given a finite-dimensional source space $S_{\Lambda_{M}}\left(B_{0}\right)$, can we identify a set of measurement frequencies $Q=\left\{k_{j}\right\}$ such that $|Q| \ll \operatorname{dim} S_{\Lambda_{M}}\left(B_{0}\right)$ and such that any $s \in S_{\Lambda_{M}}\left(B_{0}\right)$ can be reconstructed exactly from measurements at these frequencies?

2. Vice versa: Given a finite set of measurement frequencies $Q$, can we identify the finitedimensional source spaces $S_{\Lambda_{M_{j}}}\left(B_{0}\right)$ in which the sources can be reconstructed exactly from measurements at these frequencies?

3. Will such reconstructions be stable with respect to imperfect or noisy measurements? By 'stable' we mean in general that the noise in the measurements is not unacceptably amplified in the reconstructed source. More precisely, for/a fixed frequency $k$, let $U_{k}^{\varepsilon}=U_{k}+\varepsilon$ be a noisy measurement, where $\varepsilon \in L^{2}(\partial B)$ represents an additive noise term. In our reconstruction method, we will need to compute certain functionals of the measurements of the form

$$
\hat{U}=\frac{\left(U_{k}^{\varepsilon}, v_{j}\right)_{L^{2}(\partial B)}}{\sigma_{j}^{k}}=\frac{\left(U_{k}, v_{j}\right)_{L^{2}(\partial B)}}{\sigma_{j}^{k}}+\frac{\left(\varepsilon, v_{j}\right)_{L^{2}(\partial B)}}{\sigma_{j}^{k}} .
$$

The noise component in this expression will be amplified by the factor $1 / \sigma_{j}^{k}$, which is is unbounded as $j \rightarrow \infty$. Our reconstruction method is stable because we control the maximum value of $1 / \sigma_{j}^{k}$ used in the reconstruction to avoid unacceptable amplification of such additive noise.

The main results, and answers to the above questions, are summarized as follows.

Theorem 3. Let $s \in S_{\Lambda_{M}}\left(B_{0}\right)$, for some finite set $\Lambda_{M}$ of eigenvalues. There is a $\Delta k \in$ $\left(0,1 / R_{0}\right]$ such that, if for each $\lambda_{\alpha, \beta} \in \Lambda_{M}$ there is a measurement frequency $k_{j} \in Q$ satisfying $\left|k_{j}-\lambda_{\alpha, \beta}^{1 / 2}\right|<\Delta k$, then the source $s$ can be stably reconstructed from the set of measurements $\left\{U_{k_{j}}\right\}_{k_{j} \in Q}$.

Corollary 1. Let $Q$ be a finite set of measurement frequencies. There is a $\Delta k \in\left(0,1 / R_{0}\right]$ such that for any set of eigenvalues $\Lambda_{M_{j}}$ satisfying $\min _{k_{j} \in Q}\left|\lambda_{\alpha, \beta}-k_{j}\right|<\Delta k$ for all $\lambda_{\alpha, \beta} \in$ $\Lambda_{M_{j}}$, any source $s \in S_{\Lambda_{M_{j}}}\left(B_{0}\right)$ can be stably reconstructed from the measurements $\left\{U_{k_{j}}\right\}_{k_{j} \in Q}$.

\section{Remarks}

- Theorem 3 says that if we have measurements at a set of frequencies $Q$ that is close enough to the set $\Lambda_{M}$ of eigenvalues, then any source $s \in S_{\Lambda_{M}}\left(B_{0}\right)$ can be stably reconstructed from these measurements. Conversely, given a set $Q$ of measurement frequencies, we can find sets of eigenvalues satisfying the hypothesis in Theorem 3 . These sets then give rise to finite-dimensional source spaces, the elements of which are stably reconstructible. We will later also show that, due to the distribution of the 
eigenvalues of the Dirichlet Laplacian on $B_{0}$, a single frequency $k$ can be close to multiple eigenvalues. Consequently, sources from high-dimensional $S_{\Lambda_{M}}\left(B_{0}\right)$ are reconstructible even from quite few measurement frequencies.

- If $s \notin S_{\Lambda_{M}}\left(B_{0}\right)$, we reconstruct an approximation $\tilde{s}=P_{S_{\Lambda_{M}}\left(B_{0}\right)} s$, the projection of $s$ onto $S_{\Lambda_{M}}\left(B_{0}\right)$. The quality of the approximation depends on $\|\tilde{s}-s\|_{L^{2}\left(B_{0}\right)}$, and hence on $S_{\Lambda_{M}}\left(B_{0}\right)$.

- Given $S_{\Lambda_{M}}\left(B_{0}\right)$, we can extract a (not necessarily unique) smallest set of measurement frequencies $Q_{m}$ sufficient for any $s \in S_{\Lambda_{M}}\left(B_{0}\right)$ to be stably reconstructed from measurements $\left\{U_{k_{j}}\right\}_{k_{j} \in Q_{m}}$. However, our analysis does not provide a necessary criterion for such a set $Q_{m}$, and hence it might be possible to reconstruct sources from even smaller frequency sets.

The above results are achieved by utilizing the special relationship between the singular vectors of the measurement operator $F_{k}$ and the eigenfunctions of the Dirichlet-Laplacian, summarized in the following lemma.

Lemma 3. For any positive measurement frequency $k$ we have

$$
\left(\varphi_{\alpha, \beta, \gamma}, u_{m, n}^{k}\right)_{L^{2}\left(B_{0}\right)}=\delta_{\alpha, m} \delta_{\gamma, n} \operatorname{sign}\left(j_{m+1}\left(\mu_{m, \beta}\right)\right) \frac{\sqrt{8 k / \pi R_{0}} j_{m}\left(\kappa_{0}\right)}{a_{m}\left(\kappa_{0}\right)} \frac{\lambda_{m, \beta}^{1 / 2}}{\lambda_{m, \beta}-k^{2}},
$$

where $\delta_{m, n}$ is the Kronecker delta. In addition, if $k=\lambda_{m, i}^{1 / 2}$ for some $m, i$, then the rightsingular vectors of the measurement operator $F_{k}$ are the eigenvectors of the Dirichlet Laplacian: $u_{m, n}^{k}=\varphi_{m, i, n}$, for each $n \in[-m, \ldots, m]$.

Proof. From the orthonormality of the spherical harmonics, we immediately have that

$$
\left(\varphi_{\alpha, \beta, \gamma}, u_{m, n}^{k}\right)_{L^{2}\left(B_{0}\right)}=\delta_{\alpha, m} \delta_{\gamma, n} \frac{\sqrt{8 k / \pi R_{0}^{5}}}{\left|j_{\alpha+1}\left(\mu_{\alpha, \beta}\right)\right| a_{m}\left(\kappa_{0}\right)} \int_{0}^{R_{0}} j_{\alpha}\left(\mu_{\alpha, \beta} r / R_{0}\right) j_{m}(k r) r^{2} \mathrm{~d} r .
$$

Setting $\alpha=m$, evaluating the above integral and using the recurrence relations for Bessel functions, as well as that $\lambda_{\alpha, \beta}=\left(\mu_{\alpha, \beta} / R_{0}\right)^{2}$, we get

$$
\int_{0}^{R_{0}} j_{m}\left(\mu_{m, \beta} / R_{0} r\right) j_{m}(k r) r^{2} \mathrm{~d} r=\frac{R_{0}^{2} \lambda_{m, \beta}^{1 / 2}}{\lambda_{m, \beta}-k^{2}} j_{m}\left(k R_{0}\right) j_{m+1}\left(\mu_{m, \beta}\right) .
$$

This shows the first statement. For the second part we note that, when $z=\mu_{m, i}$, we have

$$
a_{m}(z)=\sqrt{\frac{2 z}{\pi}}\left(j_{m}^{2}(z)+j_{m+1}^{2}(z)-\frac{2 m+1}{z} j_{m}(z) j_{m+1}(z)\right)^{1 / 2}=\sqrt{\frac{2 z}{\pi}}\left|j_{m+1}(z)\right| .
$$

Recall that $\lambda_{m, i}^{1 / 2}=\mu_{m, i} / R_{0}$ and $\kappa_{0}=k R_{0}$. Taking $k=\lambda_{m, i}^{1 / 2}$, we have that

$$
\begin{aligned}
u_{m, n}^{k}(x) & =\frac{2 \sqrt{k / \pi}}{R_{0} a_{m}\left(\kappa_{0}\right)} j_{m}(k|x|) Y_{m}^{n}(x /|x|) \\
& =\frac{\sqrt{2}}{R_{0}^{3 / 2}\left|j_{m+1}\left(\mu_{m, i}\right)\right|} j_{m}(k|x|) Y_{m}^{n}(x /|x|) \\
& =\varphi_{m, i, n}(x), \quad m \in \mathbf{N}_{0}, n \in\{-m, \ldots, m\} .
\end{aligned}
$$


To prove Theorem 3 we also need the following result.

Lemma 4. For any numbers $k_{1}, \ldots, k_{N}$ satisfying $0<k_{1}<\cdots<k_{N}$ there exists a largest $\Delta k>0$ such that for any $1 \leq i \leq N$ and any real $x,\left|k_{i}-x\right|<\Delta k$ implies

$$
\left|\frac{k_{i}}{k_{i}^{2}-x^{2}}\right|>\sum_{j=1, j \neq i}^{N}\left|\frac{k_{j}}{k_{j}^{2}-x^{2}}\right| .
$$

Proof. For any index $i$, assume $x \in\left[k_{i}-\Delta k, k_{i}+\Delta k\right] \subset\left(k_{i-1}, k_{i+1}\right)$, with the convention that $k_{0}=0$ and $k_{N+1}>k_{N}$. The function

$$
S(x)=\sum_{j=1, j \neq i}^{N}\left|\frac{k_{j}}{k_{j}^{2}-x^{2}}\right|, \quad x \in\left[k_{i}-\Delta k, k_{i}+\Delta k\right]
$$

is convex and has a maximum $M$ at one of the endpoints $k_{i} \pm \Delta k$. Next, the function

$$
D(x)=\left|\frac{k_{i}}{k_{i}^{2}-x^{2}}\right|, \quad x \in\left[k_{i}-\Delta k, k_{i}+\Delta k\right],
$$

is unbounded at $k_{i}$, and it is even. We can therefore find a largest $\Delta k$ such that for all $x \in\left(k_{i}-\Delta k, k_{i}+\Delta k\right)$, we have $D(x)>M \geq S(x)$.

We now construct the mapping between the multi-frequency measurements and the finitedimensional source space. Let $\Lambda_{M}$ be a set of $M$ eigenvalues and $Q$ a finite set of measurement frequencies, with $|Q| \leq M$. For the sake of bookkeeping, we introduce some new notation.

1. The auxiliary frequency set $Q_{M}$ : For each $\lambda_{\alpha, \beta} \in \Lambda_{M}$, take the corresponding $k_{\alpha, \beta} \in Q_{M}$ to be $k_{\alpha, \beta}=\operatorname{argmin}_{k_{j} \in Q}\left|\lambda_{\alpha, \beta}^{1 / 2}-k_{j}\right|$, i.e., the measurement frequency closest to $\lambda_{\alpha, \beta}^{1 / 2}$. Then $Q_{M}$ is a set of $M$ not necessarily distinct numbers from $Q$.

2. We label a measurement at frequency $k_{\alpha, \beta} \in Q_{M} \subset Q$ as $U_{\alpha, \beta}$. That is, if $k_{\alpha, \beta}=k_{j} \in Q$, then $U_{\alpha, \beta}=U_{k_{j}}$.

3. Let $I_{\Lambda_{M}}$ be the increasingly ordered set of indices $\alpha$ such that $\lambda_{\alpha, \beta} \in \Lambda_{M}$. For each $\alpha \in I_{\Lambda_{M}}$, we define the set $I_{\alpha}=\left\{\beta^{\prime} \in \mathbf{N}: \lambda_{\alpha, \beta^{\prime}} \in \Lambda_{M}\right\}$, also increasingly ordered.

If for example $\Lambda_{4}=\left\{\lambda_{1,2}, \lambda_{3,1}, \lambda_{3,3}, \lambda_{7,3}\right\}$, we have that $I_{\Lambda_{4}}=\{1,3,7\}$, and that $I_{1}=\{2\}, I_{3}=\{1,3\}$ and $I_{7}=\{3\}$.

4. We associate to each measurement $U_{\alpha, \beta}$ a set of coefficients:

$$
\hat{u}_{\alpha, \beta, \gamma}=\frac{\left(U_{\alpha, \beta}, v_{\alpha, \gamma}^{k_{\alpha, \beta}}\right)_{L^{2}(\partial B)}}{\sigma_{\alpha}^{k_{\alpha, \beta}}}, \quad k_{\alpha, \beta} \in Q_{M}, \gamma \in\{-\alpha, \ldots, \alpha\}
$$

Here $v_{\alpha, \beta}^{k_{\alpha, \beta}}$ and $\sigma_{\alpha}^{k_{\alpha, \beta}}$ are the singular vectors and singluar values of $F_{k_{\alpha, \beta}}$, respectively.

We now construct a family of matrix operators mapping the eigenfunction expansion coefficients $\left\{\hat{s}_{\alpha, \beta, \gamma}\right\}$ of a source $s \in S_{\Lambda_{M}}\left(B_{0}\right)$ to the measurement coefficients $\left\{\hat{u}_{\alpha, \beta, \gamma}\right\}$. 
Expressing $s \in S_{\Lambda_{M}}\left(B_{0}\right)$ in the above notation we have

$$
s=\sum_{\alpha \in I_{\Lambda_{M}}} \sum_{\beta \in I_{\alpha}} \sum_{\gamma=-\alpha}^{\alpha} \hat{s}_{\alpha, \beta, \gamma} \varphi_{\alpha, \beta, \gamma} .
$$

Fixing $m \in I_{\Lambda_{M}}$ and letting $U_{m, n}$ be the measurement associated with the source $s$ yields

$$
\begin{aligned}
\hat{u}_{m, n, i} & =\frac{\left(U_{m, n}, v_{m, i}^{k_{m, n}}\right)_{L^{2}(\partial B)}}{\sigma_{m}^{k_{m, n}}} \\
& =\left(s, u_{m, i}^{k_{m, n}}\right)_{L^{2}\left(B_{0}\right)} \\
& =\sum_{\beta \in I_{m}} \hat{s}_{m, \beta, i}\left(\varphi_{m, \beta, i}, u_{m, i}^{k_{m, n}}\right)_{L^{2}\left(B_{0}\right)}, \quad n \in I_{m}, i \in\{-m, \ldots, m\} .
\end{aligned}
$$

This is a linear system relating the source coefficients with the measurement coefficients. Setting $N=\left|I_{m}\right|$, we define

$$
\begin{aligned}
\bar{U}_{m}^{i} & =\left(\hat{u}_{m, j, i}\right)_{j \in I_{m}} \in \mathbf{C}^{N}, \\
\bar{S}_{m}^{i} & =\left(\hat{s}_{m, j, i}\right)_{j \in I_{m}} \in \mathbf{C}^{N}, \\
K_{m} & =\left(\left(\varphi_{m, j, i}, u_{m, i}^{k_{m}, n}\right)_{L^{2}\left(B_{0}\right)}\right)_{n, j \in I_{m}} \in \mathbf{C}^{N \times N} .
\end{aligned}
$$

Notice that the matrix $K_{m}$ does not depend on the index $i$. Equation (21) now takes the form

$$
\bar{U}_{m}^{i}=K_{m} \bar{S}_{m}^{i}, \quad i \in\{-m, \ldots, m\} .
$$

To reconstruct all the coefficients of $s \in S_{\Lambda_{M}}\left(B_{0}\right)$, we thus have to solve the system of equations

$$
\bar{U}_{m}^{i}=K_{m} \bar{S}_{m}^{i}, \quad \forall m \in I_{\Lambda_{M}}, i \in\{-m, \ldots, m\},
$$

and so we have to show that the matrices $K_{m}$ are invertible.

To this end, we show that there exists a $\Delta k>0$ such that if for each $\lambda_{\alpha, \beta} \in \Lambda_{M}$ there is a corresponding measurement frequency $k_{\alpha, \beta} \in Q_{M} \subset Q$ close to $\lambda_{\alpha, \beta}$ in the sense that $\left|\lambda_{\alpha, \beta}^{1 / 2}-k_{\alpha, \beta}\right|<\Delta k$, then each matrix $K_{m}$ is invertible. In addition, we show that if $\Delta k \leq 1 / R_{0}$, then the inversion is stable with respect to noisy measurements.

Invertibility of the matrices $K_{m}$ is shown by proving that $\Delta k$ can be chosen such that each matrix $K_{m}$ is strictly diagonally dominant. Recall that an $M \times M$ matrix $A=\left(a_{i, j}\right)$ is strictly diagonally dominant if $\left|a_{i, i}\right|>\sum_{j \neq i}\left|a_{i, j}\right|$ for each $1 \leq i \leq M$, and that such matrices are invertible 21].

Proof of Theorem 3, (reconstruction): From equation (24) we get that for $K_{m}, m \in I_{\Lambda_{M}}$ to be strictly diagonally dominant, the inequality

$$
\left|\left(\varphi_{m, n, i}, u_{m, i}^{k_{m, n}}\right)_{L^{2}\left(B_{0}\right)}\right|>\sum_{\beta \in I_{m}, \beta \neq n}\left|\left(\varphi_{m, \beta, i}, u_{m, n}^{k_{m, n}}\right)_{L^{2}\left(B_{0}\right)}\right|
$$


has to hold for each row. Recall (21) and that the index $n \in I_{m}$ is row-dependent. With the help of Lemma 3 we write (27) as

$$
\left|\frac{\sqrt{8 k / \pi R_{0}} j_{m}\left(\kappa_{0}\right)}{a_{m}\left(\kappa_{0}\right)} \frac{\lambda_{m, n}^{1 / 2}}{\lambda_{m, n}-k_{m, n}^{2}}\right|>\sum_{\beta \in I_{m}, \beta \neq n}\left|\frac{\sqrt{8 k / \pi R_{0}} j_{m}\left(\kappa_{0}\right)}{a_{m}\left(\kappa_{0}\right)} \frac{\lambda_{m, \beta}^{1 / 2}}{\lambda_{m, \beta}-k_{m, n}^{2}}\right| .
$$

The factor $\sqrt{8 k / \pi R_{0}} j_{m}\left(\kappa_{0}\right) / a_{m}\left(\kappa_{0}\right)$ is constant in each row and can be neglected. Hence the simplification

$$
\left|\frac{\lambda_{m, n}^{1 / 2}}{\lambda_{m, n}-k_{m, n}^{2}}\right|>\sum_{\beta \in I_{m}, \beta \neq n}\left|\frac{\lambda_{m, \beta}^{1 / 2}}{\lambda_{m, \beta}-k_{m, n}^{2}}\right| .
$$

For fixed $m$ and increasing $\beta \in I_{m}$, the eigenvalues $\left\{\lambda_{m, \beta}\right\}_{\beta \in I_{m}}$ form a strictly increasing sequence. Assuming that $k_{m, n} \in\left(\lambda_{m, n-1}^{1 / 2}, \lambda_{m, n+1}^{1 / 2}\right)$, Lemma 4 shows that there exists a largest $\Delta k_{m, n}>0$ such that if $\left|\lambda_{m, n}^{1 / 2}-k_{m, n}\right|<\Delta k_{m, n}$ then the inequality (29) holds. We take $\Delta k_{m}$ to be $\Delta k_{m}=\min _{n \in I_{m}} \Delta k_{m, n}$, i.e., the smallest of the $\Delta k_{m, n}$ 's such that 290 holds for each row in $K_{m}$. Next, we set $\Delta k=\min _{m \in I_{\Lambda_{M}}}$. Assume now that for each $\lambda_{m, n} \in \Lambda_{M}$ there is a measurement frequency $k_{j} \in Q$ such that $\left|\lambda_{m, n}^{1 / 2}-k_{j}\right|<\Delta k$. Then, for $k_{m, n}=k_{j} \in Q_{M}$, we immediately have that

$$
\left|\lambda_{m, n}^{1 / 2}-k_{m, n}\right|<\Delta k \leq \Delta k_{m, n}
$$

and hence the inequality 29 holds for each row in each $K_{m}$. Hence each $K_{m}$ is strictly diagonally dominant and invertible.

\section{Remark}

The above proof finds a single $\Delta k$ that serves as a "global" constraint on how close it suffices for the measurement frequencies to be to the eigenvalues in $\Lambda_{M}$. This is done to make the statement of Theorem 3 simpler. In a more refined analysis, one could aim to find a $\Lambda_{M}$-dependent $\Delta k$. Moreover, the above is merely an existence proof for $\Delta k$. Clearly, we want $\Delta k$ as large as possible (but, as we will see, smaller than or equal to $1 / R_{0}$ for stability reasons). This can of course be checked numerically, by computing the rank or condition number of the matrices $K_{m}$ as a function of $\Delta k$, and doing this reveals that one can choose $\Delta k=1 / R_{0}$. However, we have not yet been able to obtain a proof of this.

We now analyze the stability of the above inversion procedure with respect to noise. We will see that under the simple constraint that $\Delta k \leq 1 / R_{0}$, where $R_{0}$ is the radius of the source domain, we can guarantee stable reconstructions in the sense that noise is not amplified in the calculation of the measurement coefficients $\hat{u}_{m, n, i}$.

For $\varepsilon \in L^{2}(\partial B)$, we consider an additive noise model where the noisy measurements are of the form $U_{m, n}^{\varepsilon}=U_{m, n}+\varepsilon$. We assume the noise $\varepsilon$ to be bounded in the sense that $\|\varepsilon\|_{L^{2}(\partial B)} \unlhd \delta\left\|U_{m, n, i}\right\|_{L^{2}(\partial B)}$ for some noise level $0<\delta<1$. Recalling equation (20) for the measurement coefficients,

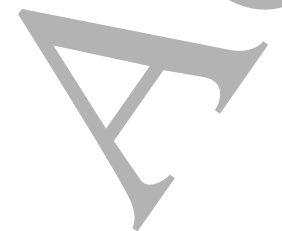

$$
\hat{u}_{m, n, i}=\frac{\left(U_{m, n}^{\varepsilon}, v_{m, i}^{k_{m, n}}\right)_{L^{2}(\partial B)}}{\sigma_{m}^{k_{m, n}}}=\frac{\left(U_{m, n}, v_{m, i}^{k_{m, n}}\right)_{L^{2}(\partial B)}}{\sigma_{m}^{k_{m, n}}}+\frac{\left(\varepsilon, v_{m, i}^{k_{m, n}}\right)_{L^{2}(\partial B)}}{\sigma_{m}^{k_{m, n}}},
$$



$\lambda_{m, n}=\left(\mu_{m, n} / R_{0}\right)^{2}$, we have that

$$
\left|\lambda_{m, n}^{1 / 2}-k_{j}\right|<\Delta k \Longleftrightarrow \mu_{m, n} \in\left(k_{j} R_{0}-1, k_{j} R_{0}+1\right) .
$$

Assume first that the largest index $\widetilde{m} \in I_{k_{j}}$ is associated with some $\mu_{\widetilde{m}, 1}$ satisfying

$$
\mu_{\tilde{m}, 1} \in\left(k_{j} R_{0}-1, k_{j} R_{0}+1\right) .
$$

Then either $\mu_{\widetilde{m}, 1}<k_{j} R_{0}$ and so $\widetilde{m}<\mathscr{B}_{-}$, or $\mu_{\widetilde{m}, 1} \in\left[k_{j} R_{0}, k_{j} R_{0}+1\right)$ and so $\widetilde{m}=\mathscr{B}_{-}$, since no two $\mu_{m, 1}$ can occur within an interval of length 1 , and $\mu_{\mathscr{B}_{-}, 1}$ is the smallest $\mu_{m, 1} \geq k_{j} R_{0}$. Last, assume the largest index $\widetilde{m}$ comes from $\mu_{\widetilde{m}, n}, n>1$, and that $\widetilde{m}>\mathscr{B}_{-}$. Then $\mu_{\widetilde{m}, n}>\mu_{\widetilde{m}, 1}>\mu_{\mathscr{B}_{-}, 1}$, which is a contradiction since then $\mu_{\widetilde{m}, n} \notin\left(k_{j} R_{0}-1, k_{j} R_{0}+1\right)$.

\subsubsection{Density of eigenvalues and subspace dimension}

A key observation in [15] was that the eigenvalues become more densely spaced as one moves up the real line. An estimate for the expected number $N_{\lambda}(k, \Delta k)$ of $\lambda_{\alpha, \beta}^{1 / 2}$ that lay in an interval of length $2 \Delta k$ centered at $k$ was in 15 given by the formula

$$
N_{\lambda}(k, \Delta k) \approx\left\lfloor\left(\frac{1}{2}\left(3 k^{2}+16\right)^{1 / 2}-3\right)\right\rfloor \frac{2 \Delta k}{R_{0} \pi}
$$

Roughly, this implies that for a set of $N$ linearly spaced frequencies $Q=\{2 \Delta k \cdot n\}_{n=1}^{N}$, we have

$$
|\Lambda| \approx \frac{(\Delta k)^{2}}{R_{0} \pi} 3 N(N+1),
$$

and hence that the cardinality of $\Lambda_{M}$ increases approximately quadratically with $N$. Equation (31) also has the interpretation that high-frequency measurements carry more information in terms of the dimension of $S_{\Lambda_{M}}\left(B_{0}\right)$. 
For numerical experiments and validation of the above reconstruction method, we introduce two finite-dimensional source spaces. Let $\Lambda_{L}=\left\{\lambda_{\alpha, \beta}\right\}_{\alpha=0, \beta=1}^{5,5}$ and $\Lambda_{H}=\left\{\lambda_{\alpha, \beta}\right\}_{\alpha=0, \beta=1}^{15,15}$, and let $S_{\Lambda_{L}}$ and $S_{\Lambda_{H}}$ be the corresponding source spaces. Further, let the source support $B_{0}$ have radius 1 , and assume measurements are taken on $\partial B$, where $B$ has radius 2 . By numerical testing, we find that we can choose $\Delta k=0.7$ in Theorem 3 and we find the smallest (non-unique) sets of measurement frequencies $Q_{L}$ and $Q_{H}$ sufficient for stable reconstruction of sources in $S_{\Lambda_{L}}$ and $S_{\Lambda_{H}}$, respectively. We get

$$
\begin{aligned}
& \left|Q_{L}\right|=12, \quad\left|\Lambda_{L}\right|=30, \quad \operatorname{dim} S_{\Lambda_{L}}=180, \\
& \left|Q_{H}\right|=42, \quad\left|\Lambda_{H}\right|=240, \quad \operatorname{dim} S_{\Lambda_{H}}=3840 .
\end{aligned}
$$

Due to the density of the eigenvalues, we see that we can reconstruct 3840 source coefficients from measurements at only 42 different frequencies.

Let $\chi_{B_{r, x_{0}}}(x)$ be the characteristic function for a ball of radius $r$ centered at $x_{0}$ in $\mathbf{R}^{3}$. We consider the two sources

$$
\begin{aligned}
& s_{E}(x)=1 \varphi_{2,1,1}+2 \varphi_{3,3,3}+4 \varphi_{4,3,2}+0.5 \varphi_{12,6,7}, \\
& s_{B}(x)=1 \chi_{B_{r_{1}, x_{1}}}(x)+2 \chi_{B_{r_{2}, x_{2}}}(x)+3 / 2 \chi_{B_{r_{3}, x_{3}}}(x),
\end{aligned}
$$

where the radii and centers of the characteristic functions are

$$
r_{1}=0.4, x_{1}=(0,0.1,0.3), r_{2}=0.1, x_{2}=(0,-0.4,-0.3), r_{3}=0.3, x_{3}=(0.2,0.5,-0.4) .
$$

Clearly, $s_{E} \in S_{\Lambda_{H}} \backslash S_{\Lambda_{L}}$. On the other hand, $s_{B}$ belongs to neither $S_{\Lambda_{L}}$ nor $S_{\Lambda_{H}}$, and we can only reconstruct the projection of the source onto the source spaces.

To compute the measurements $\left\{U_{k_{j}}\right\}_{k_{j} \in Q}$, we do as follows:

For a source $\chi_{B_{r, x_{0}}}(x)$ supported in $B_{0}$ we have

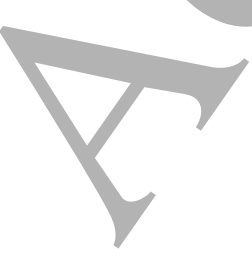

$$
\begin{aligned}
F_{k} \chi_{B_{r, x_{0}}}(x) & =\int_{B_{0}} G_{k}(x, y) \chi_{B_{r, x_{0}}}(y) \mathrm{d} y \\
& =\int_{B_{r, x_{0}}} \frac{e^{i k|x-y|}}{4 \pi|x-y|} \mathrm{d} y=\frac{1}{4 \pi} \sum_{n=0}^{\infty} \frac{(i k)^{n}}{n !} \int_{B_{r, x_{0}}}|x-y|^{n-1} \mathrm{~d} y .
\end{aligned}
$$


The integrals in the sum can be evaluated by noting that the function $|x-y|^{n-1}$ is constant for $y \in \partial B_{z, x}$. Let $d=\left|x-x_{0}\right|$. For $d-r \leq z \leq d+r$ the sphere $\partial B_{z, x}$ intersects $B_{r, x_{0}}$, and the part of $\partial B_{z, x}$ enclosed in $B_{r, x_{0}}$ is a spherical cap with area $A(z)=2 \pi z\left(z-\left(d^{2}-r^{2}+z^{2}\right) / 2 d\right)$, see [24]. We thus have that

$$
\int_{B_{r, x_{0}}}|x-y|^{n-1} \mathrm{~d} y=\int_{d-r}^{d+r} z^{n-1} 2 \pi z\left(z-\frac{d^{2}-r^{2}+z^{2}}{2 d}\right) \mathrm{d} z .
$$

This integral can be evaluated by standard methods and we get

$$
\int_{B_{r, x_{0}}}|x-y|^{n-1} \mathrm{~d} y=\frac{2 \pi\left((d+r(2+n))(d-r)^{n+2}+((n+2) r-d)(d+r)^{n+2}\right)}{d(n+1)(n+2)(n+3)} .
$$

A lengthy calculation shows that

$$
\begin{aligned}
& \sum_{n=0}^{\infty} \frac{(i k)^{n}}{4 \pi} \frac{2 \pi\left((d+r(2+n))(d-r)^{n+2}+((n+2) r-d)(d+r)^{n+2}\right)}{d n !(n+1)(n+2)(n+3)} \\
& =\frac{(i-k r) \mathrm{e}^{i k(d-r)}-(i+k r) \mathrm{e}^{i k(d+r)}}{2 d k^{3}},
\end{aligned}
$$

and hence the measurement is given by

$$
F_{k} \chi_{B_{r, x_{0}}}(x)=\frac{(i-k r) \mathrm{e}^{i k\left(\left|x-x_{0}\right|-r\right)}-(i+k r) \mathrm{e}^{i k\left(\mid x^{\left.-x_{0} \mid+r\right)}\right)}}{2\left|x-x_{0}\right| k^{3}}, \quad x_{0} \in B_{0}, x \in \partial B .
$$

The measurement corresponding to an eigenvector source $\varphi_{\alpha, \beta, \gamma}$ is given by 19 in Lemma 3, and we have that

$$
F_{k} \varphi_{\alpha, \beta, \gamma}(x)=\sigma_{\alpha}^{k} v_{\alpha, \gamma}^{k}(x) \operatorname{sign}\left(j_{\alpha+1}\left(\mu_{\alpha, \beta}\right)\right) \frac{\sqrt{8 k / \pi R_{0}} j_{\alpha}\left(\kappa_{0}\right)}{a_{\alpha}\left(\kappa_{0}\right)} \frac{\lambda_{\alpha, \beta}^{1 / 2}}{\lambda_{\alpha, \beta}-k^{2}} .
$$

We avoid the high computational cost of simulating high-frequency solutions to the Helmholtz equation by generating the measurements of the sources using the above closed-form solutions. We sample each of the measurements $\left\{U_{k_{j}}\right\}_{k_{j} \in Q}$ at equidistant points $\left\{x_{i}\right\}_{i=1}^{N}$ on $\partial B$. For a noise level $\delta$, we add a noise vector $\varepsilon \in \mathbf{C}^{N}$ of normally distributed random number $2^{2}$ to the measurement vector $\left(U_{k_{j}}\left(x_{i}\right)\right) \in \mathbf{C}^{N}$, with the convention that $|\varepsilon| \leq \delta\left|U_{k_{j}}\right|$. We denote the noisy measurement by $U_{k_{j}}^{\varepsilon}=U_{k_{j}}+\varepsilon$.

To compute the measurement coefficients, we approximate the inner products appearing in (21) by a simple quadrature method

$$
\left(U_{k_{j}}^{\varepsilon}, v_{\alpha, \gamma}^{k_{j}}\right)_{L^{2}(\partial B)} \approx 4 \pi R^{2} \frac{1}{N} \sum_{i=1}^{N} U_{k_{j}}^{\varepsilon}\left(x_{i}\right) \overline{v_{\alpha, \gamma}^{k_{j}}\left(x_{i}\right)}
$$

All entries in the matrices $K_{m}$ in (21) are explicitly given, and are implemented in Matlab. We compute the source coefficients by solving

$$
\bar{S}_{m}^{i}=K_{m}^{-1} \bar{U}_{m}^{i}, \quad \forall m \in I_{\Lambda}, i \in[-m, \ldots, m],
$$

where $\bar{U}_{m}^{i}$ are the vectors of measurement coefficients calculated from the noisy measurements in (40). Ultimately, the source is expanded in terms of the eigenvectors in $S_{\Lambda}$. The number of points necessary for a good estimate varies with the nature of the measurement and the dimension of the source space.

${ }^{2}$ For each component $\epsilon_{i}$ the real and imaginary part is generated separately by the Matlab-function $\operatorname{randn}()$. 


\subsection{Reconstructions}

The measurements are generated as outlined above, and we add noise with a noise level $\delta=0.1$, i.e., $10 \%$ noise to all measurements before we perform the reconstruction. We sample the measurements at $N=201$ and $N=10001$ points. We plot the $(0, y, z)$-sections of the sources, the reconstructions and their $(\log )$ pointwise absolute difference. Figures 1,3 show the results of the numerical simulations. Figures 1 and 3, made using 10001 sampling points, show that the source $s_{E}$ agrees remarkably well with the reconstruction from high-frequency measurements $\left\{U_{k_{j}}\right\}_{k_{j} \in Q_{H}}$. The outstanding agreement in the presence of noisy measurements might be due to the small number of measurement coefficients being non-zero because of the very simple structure of $s_{E}$. From the low-frequency measurements $\left\{U_{k_{j}}\right\}_{k_{j} \in Q_{L}}$ we can only reconstruct the projection $P_{S_{\Lambda_{L}}} s_{B}$, and hence we miss the component $0.5 \varphi_{12,6,7}$ of $s_{E}$. In Figure 3, we see that the reconstruction of $s_{B}$ from the low-frequency measurements captures the characteristic functions with larger supports quite well, but that the smallest ball is almost invisible. This is as expected, since $S_{\Lambda_{L}}$ does not contain the high-frequency vectors needed to resolve smaller features. On the contrary, the reconstruction of $s_{B}$ from the high-frequency measurements captures all characteristic functions quite well and is a satisfactory approximation to $s_{B}$. As we can see in the difference plots, both the low and high-frequency reconstructions suffer from larger pointwise errors around the discontinuities of $s_{B}$, and we notice the well-known Gibbs phenomenon.

The remarkable quality of the reconstructions in figures 1 and 3 is also due to significant oversampling (10001 measurement points) of noise in the measurement and the resulting improvement of the signal-to-noise ratio. To investigate the effect of oversampling on the source reconstruction, we here include figure 2. The results in that figure are obtained using only 201 sampling points, with the same noise level of $10 \%$. While these results are indeed of lower quality than the results of the oversampled case, they still seem quite good.

The results verify that the proposed reconstruction method offers stable reconstruction of sources in $S_{\Lambda}\left(B_{0}\right)$ and of projections $P_{S_{\Lambda_{L}}} s$ for $s \in L^{2}\left(B_{0}\right)$, and that the quality of such reconstructions depends on the number of measurement frequencies. Moreover, the method has a low computational cost, requiring only addition and inversion of low-dimensional matrices.

\subsection{Approximation properties of $S_{\Lambda_{M}}$}

We now study numerically the âpproximation properties of $S_{\Lambda_{M}}$. The approximation property we are interested in the distance $\left\|P_{S_{\Lambda_{M}}} s-s\right\|_{L^{2}\left(B_{0}\right)}$. We want to compare approximations of $s \in L^{2}\left(B_{0}\right)$ in $S_{\Lambda_{M}}$ and in $E_{Q}$, where $E_{Q}$ is a large set of right-singular vectors of the measurement operators $F_{k_{j}}, k_{j} \in Q$.

Let $Q=\left\{k_{j}\right\}_{j=1}^{N}$ be the smallest frequency set associated with $\Lambda_{M}$ and $s \in L^{2}\left(B_{0}\right)$. We define the truncated measurement operator at frequency $k_{j}$ by

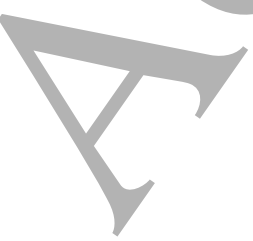

$$
F_{k_{j}}^{T}=\sum_{m=1}^{\beta_{-}^{k_{j}}} \sum_{n=-m}^{m} \sigma_{m}^{k_{j}} v_{m, n}^{k_{j}}\left(\cdot, u_{m, n}^{k_{j}}\right)_{L^{2}(\Omega)}, \quad k_{j} \in Q, \quad \beta_{-}^{k_{j}}=\operatorname{argmin}_{m \in \mathbf{N}_{0}}\left\{\mu_{m, 1} \geq k_{j} R_{0}\right\} .
$$


Next, we define the multi-frequency and truncated multi-frequency measurement operators by, respectively,

$$
F_{Q}=\left[\begin{array}{c}
F_{k_{1}} \\
F_{k_{2}} \\
\vdots \\
F_{k_{N}}
\end{array}\right], \quad F_{Q}^{T}=\left[\begin{array}{c}
F_{k_{1}}^{T} \\
F_{k_{2}}^{T} \\
\vdots \\
F_{k_{N}}^{T}
\end{array}\right]
$$

We have that $F_{Q}, F_{Q}^{T}: L^{2}\left(B_{0}\right) \rightarrow\left(L^{2}(\partial B)\right)^{N}$. Since $F_{Q}^{T}$ is finite-rank, it has a finite singular system $\left\{\sigma_{m}, \phi_{m}, \psi_{m}\right\}_{m=1}^{M}$, and the inverse of $F_{Q}^{T}$ is

$$
\left(F_{Q}^{T}\right)^{-1}=\sum_{m=1}^{M} \frac{\left(\cdot, \psi_{m}\right)_{\left(L^{2}(\partial B)\right)^{N}}}{\sigma_{m}} \phi_{m}
$$

We say that $\left(F_{Q}^{T}\right)^{-1}$ is the truncated singular value decomposition (TSVD) inverse of $F_{Q}$, and we have that $\operatorname{Im}\left(F_{Q}^{T}\right)^{-1}=\operatorname{span}\left\{\phi_{m}\right\}$. Since the inversion method using $S_{\Lambda_{M}}$ only extracts parts of the recoverable information from the measurements, it is of interest to compare it to approximations in $E_{Q}=\operatorname{span}\left\{\phi_{m}\right\}$, which in a sense is the largest space of stably recoverable information we can reconstruct from the measurements $\left\{U_{k_{j}}\right\}_{k_{j} \in Q}$.

To this end, we proceed as follows. For $k_{j} \in Q$, let $E_{k_{j}}$ be the set of right-singular vectors included in $F_{k_{j}}^{T}$, i.e.,

$$
E_{k_{j}}=\left\{u_{m, n}^{k_{j}}: 0 \leq m \leq \mathscr{B}_{-}^{k_{j}},-m \leq n \leq m\right\} .
$$

For our investigation, we need the following lemma.

Lemma 5. $\operatorname{Im}\left(F_{Q}^{T}\right)^{-1}=\operatorname{span}\left\{\bigcup_{k_{j} \in Q} E_{k_{j}}\right\}$.

Proof. First, note that $\operatorname{Ker} F_{Q}^{T}=\bigcap_{k_{j} \in Q} \operatorname{Ker} F_{k_{j}}^{T}$, and that $\operatorname{Ker} F_{k_{j}}=L^{2}(\Omega) \ominus \operatorname{span} E_{k_{j}}$. Hence

$$
\operatorname{Ker} F_{Q}^{T}=\bigcap_{k_{j} \in Q}\left(L^{2}(\Omega) \ominus \operatorname{span} E_{k_{j}}\right)=L^{2}(\Omega) \ominus \operatorname{span}\left\{\bigcup_{k_{j} \in Q} E_{k_{j}}\right\} .
$$

The result now follows by noting that

$$
\operatorname{Im}\left(F_{Q}^{T}\right)^{-1}=\left(\operatorname{Ker} F_{Q}^{T}\right)^{\perp}=\left(L^{2} \ominus \operatorname{span}\left\{\bigcup_{k_{j} \in Q} E_{k_{j}}\right\}\right)^{\perp}=\operatorname{span}\left\{\bigcup_{k_{j} \in Q} E_{k_{j}}\right\} .
$$

Hence we have $E_{Q}=\operatorname{span}\left\{\bigcup_{k_{j} \in Q} E_{k_{j}}\right\}$. To compare approximations, we compute the distances $d\left(s, S_{\Lambda_{M}}\right)$ and $d\left(s, E_{Q}\right)$ for different $s \in L^{2}\left(B_{0}\right)$ and different $\Lambda_{M}$ and $Q$.

Since $S_{\Lambda_{M}}$ is the span of orthonormal vectors,

$$
d\left(s, S_{\Lambda_{M}}\right)^{2}=\|s\|^{2}-\sum_{\varphi_{\alpha, \beta, \gamma} \in S_{\Lambda_{M}}}\left|\left(s, \varphi_{\alpha, \beta, \gamma}\right)\right|^{2} .
$$

From Chapter 7.6 in 9 , we have that the distance from a finite-dimensional subspace $M=$ $\operatorname{span}\left\{\phi_{m}\right\}_{m=1}^{M}$ of a infinite-dimensional Hilbert space $X$ to any $s \in X$ is given by the formula

$$
d(s, M)^{2}=\|s\|_{X}^{2}-\beta^{T} G^{-1} \beta .
$$


The inner products in equations (44) and (45) are approximated with the Matlab quadrature integral3(). Figure 4 shows the (relative) distances to the sources for increasing $n$ and the dimensions of $E_{Q_{n}}=\operatorname{Im}\left(F_{Q_{n}}^{T}\right)^{-1}$ and $S_{\Lambda_{M_{n}}}$. For both sources the approximation in $E_{Q_{n}}$ is slightly better than in $S_{\Lambda_{M_{n}}}$, but the dimension, and hence the computational cost associated with the reconstruction/approximation increases dramatically with $n$. Hence the results indicate that there is a large number of redundant veetors in $E_{Q_{n}}$, and that the lowerdimensional space $S_{\Lambda_{M}}$ does capture the essential information about the sources present in the multi-frequency measurements.

\section{Conclusion}

We have analyzed the feasibility and the stability of solution of the multi-frequency inverse source problem for the Helmholtz equation in $\mathbf{R}^{3}$. Given any finite set of measurement frequencies, we have characterized certain finite-dimensional source spaces in which exact source reconstruction is guaranteed if the measurements are noise-free. Conversely, for each of the finite-dimensional source spaces we have found the smallest set of measurement frequencies that is sufficient for exact reconstruction in the absence of noise. Regarding stability, we have extended our results from the two-dimensional case and proved a lower bound on the 'bandwidth' of the measurement operator, at any measurement frequency. We showed that our inversion method for multi-frequency measurements is stable and highly efficient; since the method does not rely on discretization of the measurement operator, one avoids the computational cost associated with wave phenomena in higher dimensions. It would be interesting to see if the method can be generalized to measurement boundaries other than spheres, as well as to compare its performance against commonly used inversion techniques. Finally, it remains to investigate whether also in the non-constant coefficient case a similar relationship exists between the eigenvalues and eigenvectors of an elliptic PDE and the frequency dependence of the SVD of the measurement operator.

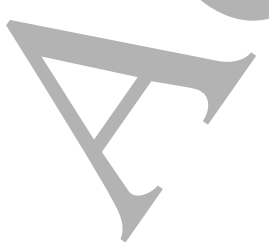




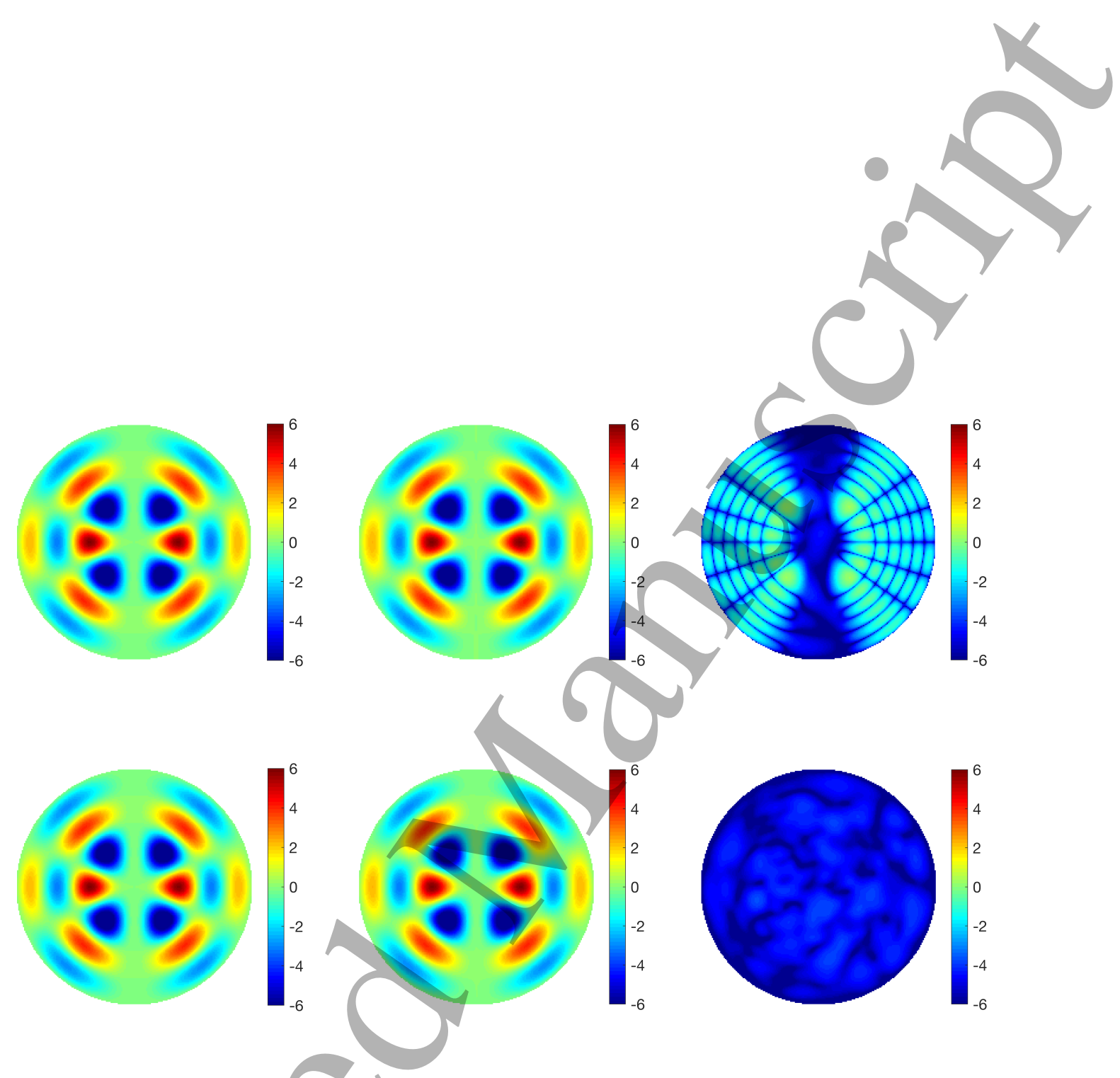

Figure 1: All plots are in the $(0, y, z)$-plane of $B_{0}$. The reconstruction is made using 10001 sampling points. Top-left: Source $s_{E}(x)$. Top-middle: Reconstruction $\hat{s}_{E}^{L}(x)$ of $s_{E}(x)$ from low-frequency measurements $\left\{U_{k_{j}}\right\}_{k_{j} \in Q_{L}}$. Top-Right: Logarithm of pointwise absolute error, $\log \left|\hat{s}_{E}^{L}(x)-s_{E}(x)\right|$. Bottom-left: Source $s_{E}(x)$. Bottom-middle: Reconstruction $\hat{s}_{E}^{H}(x)$ of $s_{E}(x)$ from high-frequency measurements $\left\{U_{k_{j}}\right\}_{k_{j} \in Q_{H}}$. Bottom-Right: Logarithm of pointwise absolute error, $\log \left|\hat{s}_{E}^{H}(x)-s_{E}(x)\right|$. 

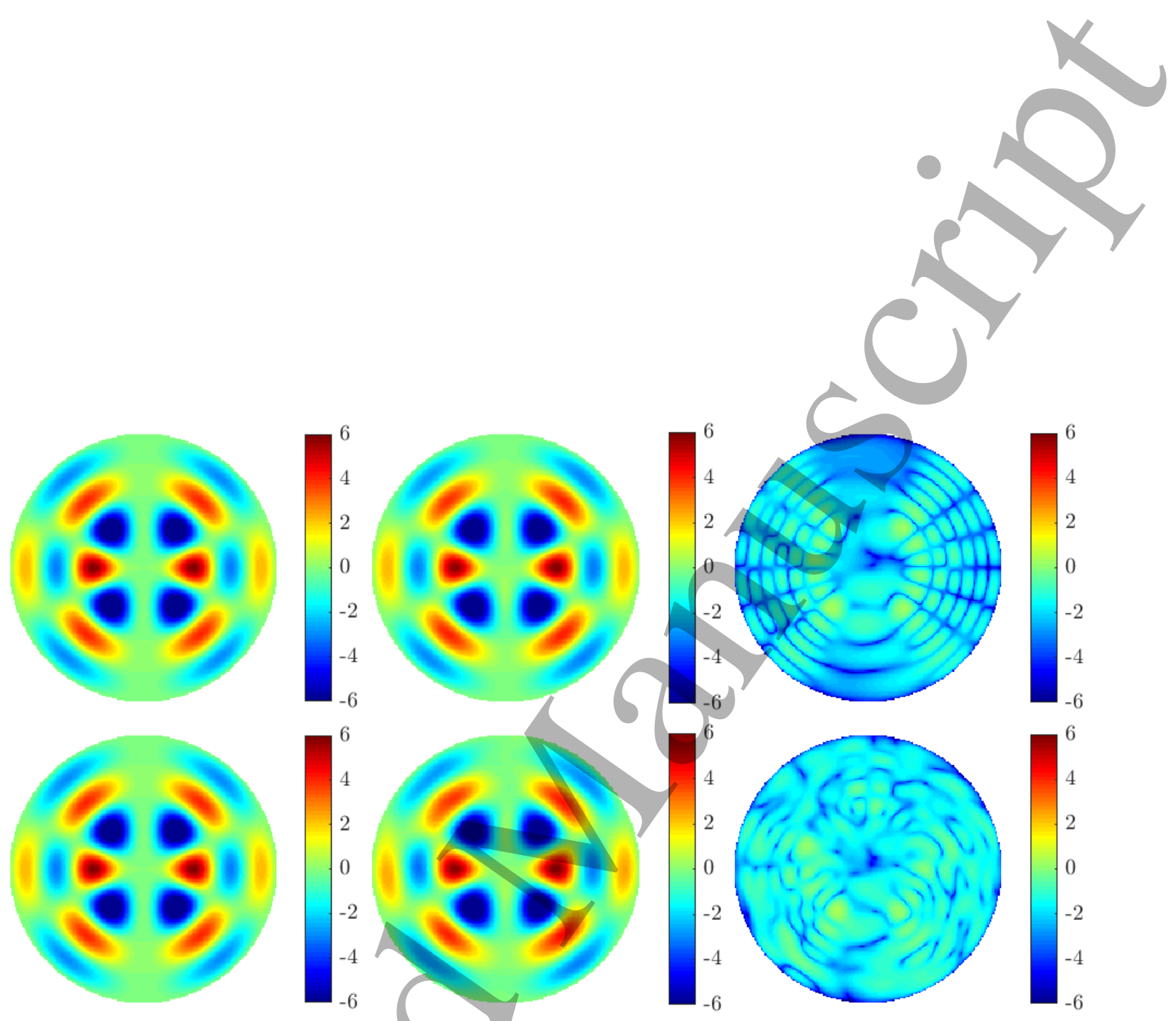

Figure 2: All plots are in the $(0, y, z)$-plane of $B_{0}$. The reconstruction is made using 201 sampling points. Top-left: Source $s_{E}(x)$. Top-middle: Reconstruction $\hat{s}_{E}^{L}(x)$ of $s_{E}(x)$ from low-frequency measurements $\left\{U_{k_{j}}\right\}_{k_{j} \in Q_{L}}$. Top-Right: Logarithm of pointwise absolute error, $\log \left|\hat{s}_{E}^{L}(x)-s_{E}(x)\right|$. Bottom-left: Source $s_{E}(x)$. Bottom-middle: Reconstruction $\hat{s}_{E}^{H}(x)$ of $s_{E}(x)$ from high-frequency measurements $\left\{U_{k_{j}}\right\}_{k_{j} \in Q_{H}}$. Bottom-Right: Logarithm of pointwise absolute error, $\log \left|\hat{s}_{E}^{H}(x)-s_{E}(x)\right|$. 


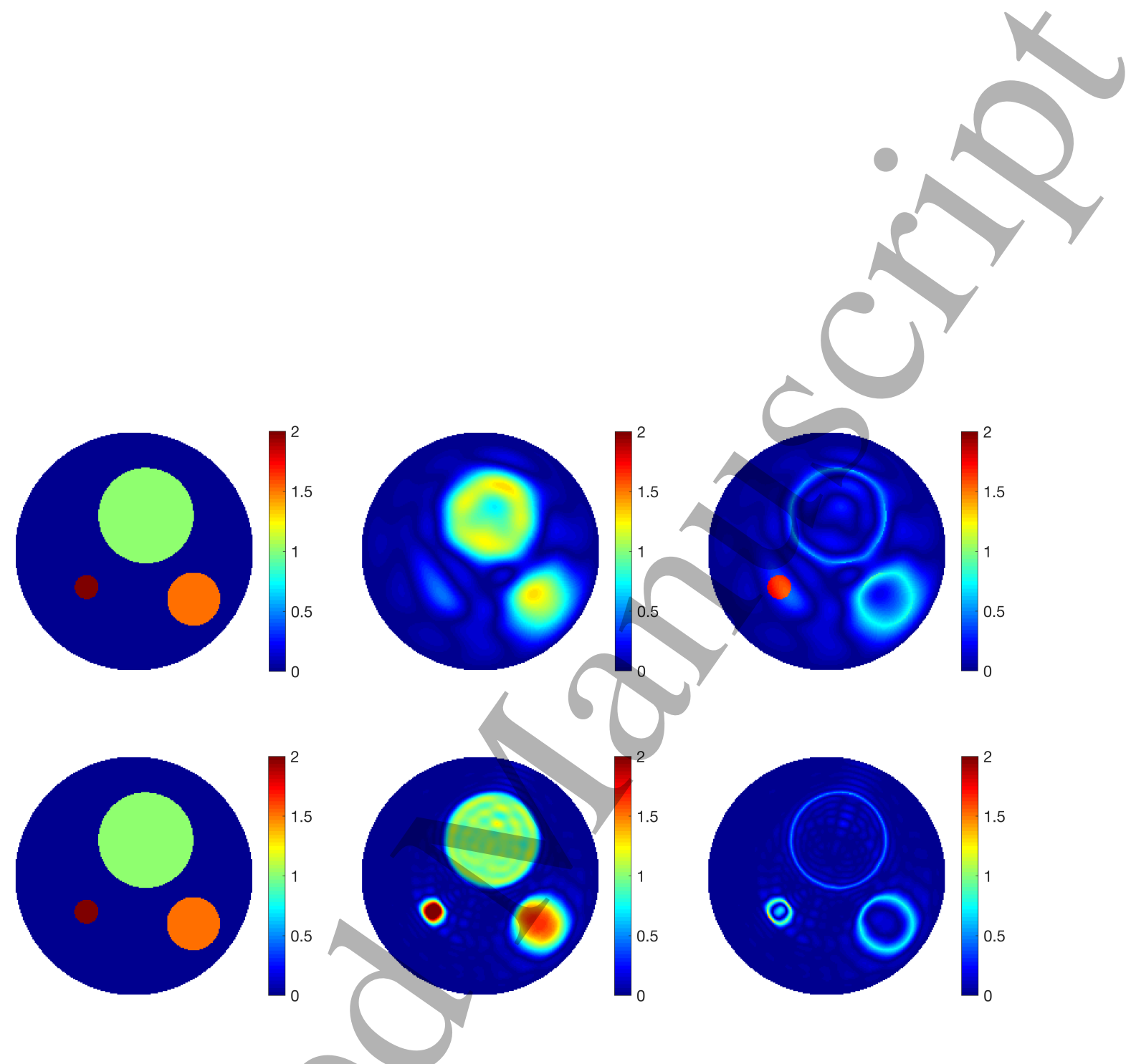

Figure 3: All plots are in the $(0, y, z)$-plane of $B_{0}$. The reconstruction is made using 10001 sampling points. Top-left: Source $s_{B}(x)$. Top-middle: Reconstruction $\hat{s}_{B}^{L}(x)$ of $s_{B}(x)$ from low-frequency measurements $\left\{U_{k_{j}}\right\}_{k_{j} \in Q_{L}}$. Top-Right: Pointwise absolute error $\left|\hat{s}_{B}^{L}(x)-s_{B}(x)\right|$. Bottom-left: Source $s_{B}(x)$. Bottom-middle: Reconstruction $\hat{s}_{B}^{H}(x)$ of $s_{B}(x)$ from high-frequency measurements $\left\{U_{k_{j}}\right\}_{k_{j} \in Q_{H}}$. Bottom-Right: Pointwise absolute error $\left|\hat{s}_{B}^{H}(x)-s_{B}(x)\right|$.

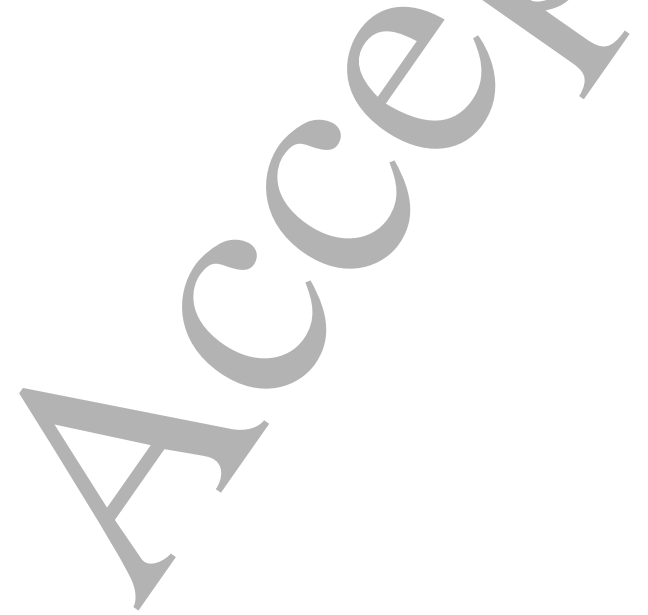




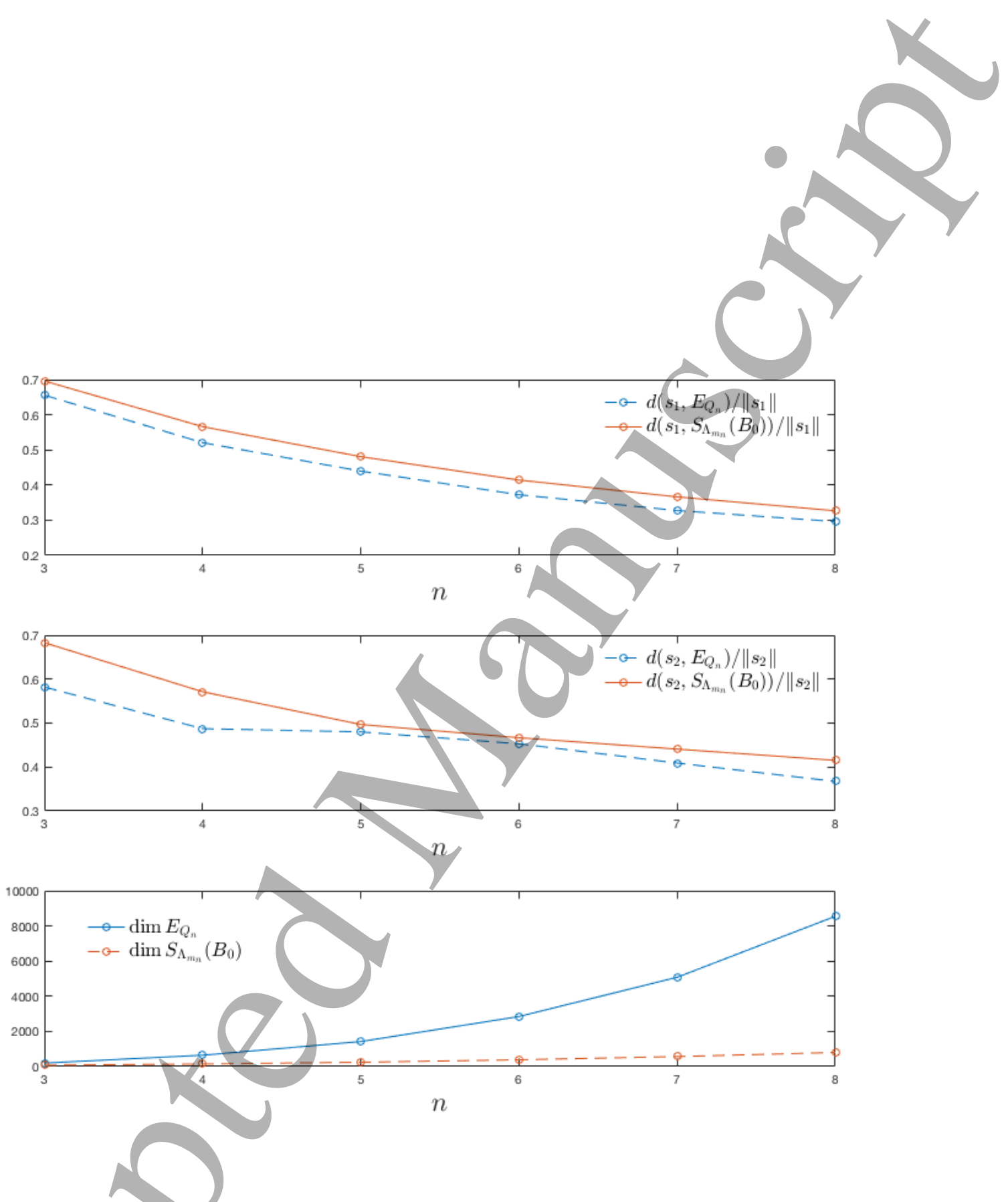

Figure 4: The top and middle plot shows the relative approximation errors in $S_{\Lambda_{M_{n}}}$ and $E_{Q_{n}}$. The bottom plot shows the dimension of the two spaces. 


\section{Bibliography}

\section{References}

[1] H Abdullah and AK Louis. The approximate inverse for solving an inverse scattering problem for acoustic waves in an inhomogeneous medium. Inverse Problems, 15(5):1213, 1999.

[2] S Acosta, S Chow, J Taylor, and V Villamizar. On the multi-frequency inverse source problem in heterogeneous media. Inverse problems, 28(7):075013, 2012.

[3] H Ammari, J Garnier, and K Sølna. Resolution and stability analysis in full-aperture, linearized conductivity and wave imaging. Proceedings of the American Mathematical Society, 141(10):3431-3446.

[4] MA Anastasio, J Zhang, D Modgil, and PJ La Rivière. Application of inverse source concepts to photoacoustic tomography. Inverse Problems, 23(6):S21, 2007.

[5] G Bao, J Lin, and F Triki. A multi-frequency inverse source problem. Journal of Differential Equations, 249(12):3443 - 3465, 2010.

[6] Gang Bao, Peijun Li, and Yue Zhao. Stability for the inverse source problems in elastic and electromagnetic waves. Journal de Mathématiques Pures et Appliquées, 2019.

[7] Gang Bao, Shuai Lu, William Rundell, and Boxi Xu. A recursive algorithm for multifrequency acoustic inverse source problems. SIAM Journal on Numerical Analysis, 53(3):1608-1628, 2015.

[8] D Colton and R Kress. Inverse acoustic and electromagnetic scattering theory, volume 93. Springer Science \& Business Media, 3rd edition, 2013.

[9] FR Deutsch. Best approximation in inner product spaces. Springer Science \& Business Media, 2012.

[10] Grigoriu Ilich Eskin. Lectures on linear partial differential equations, volume 123. American Mathematical Soc., 2011.

[11] M Fink and C Prada. Acoustic time-reversal mirrors. Inverse Problems, 17(1):R1-R38, 2001.

[12] R Griesmaier, M Hanke, and T Raasch. Inverse source problems for the Helmholtz equation and the windowed Fourier transform. SIAM Journal on Scientific Computing, 34(3):A1544-A1562, 2012.

[13] M Idemen and A Alkumru. On an inverse source problem connected with photo-acoustic and thermo-acoustic tomographies. Wave Motion, 49(6):595-604, 2012.

[14] M Karamehmedović. Explicit tight bounds on the stably recoverable information for the inverse source problem. Journal of Physics Communications, 2:095021, 2018. 
[15] M Karamehmedović, A Kirkeby, and K Knudsen. Stable source reconstruction from a finite number of measurements in the multi-frequency inverse source problem. Inverse Problems, 34(6):065004, 2018.

[16] G A McMechan, J H Luetgert, and W D Mooney. Imaging of earthquake sources in Long Valley Caldera, California, 1983. Bull. Seismol. Soc. Am., 75(4):1005-1020, 1985.

[17] Griesmaier R and Sylvester J. Far field splitting by iteratively reweighted $l^{1}$ minimization. SIAM Journal of Applied Mathematics, 76(2):705-730, 2016.

[18] Griesmaier R and Sylvester J. Uncertainty principles for inverse source problems, far field splitting, and data completion. SIAM Journal of Applied Mathematics, 77(1):154-180, 2017.

[19] Griesmaier R, Hanke M, and Sylvester J. Far field splitting for the Helmholtz equation. SIAM Journal of Numerical Analysis, 52(1):343-362, 2014.

[20] WA Strauss. Partial differential equations, volume 92. Wiley New York, 1992.

[21] RS Varga. Gershgorin and His Circles in Springer Series in Computational Mathematics, 36. Springer, Berlin, 2004.

[22] X Wang, Y Guo, D Zhang, and H Liu. Fourier method for recovering acoustic sources from multi-frequency far-field data. Inverse Problems, 33(3):035001, 2017.

[23] GN Watson. A treatise on the theory of Bessel functions. Cambridge university press, 1995.

[24] Eric W. Weisstein. Sphere-sphere intersection. From MathWorld-A Wolfram Web Resource.

[25] Deyue Zhang and Yukun Guo. Fourier method for solving the multi-frequency inverse source problem for the Helmholtz equation. Inverse Problems, 31(3):035007, 2015. 